\title{
Josephson Effect and Associated Phenomena in Double Layer Quantum Hall Systems
}

\author{
Z. F. Ezawa \\ Department of Physics, Tohoku University, Sendai, 980 Japan \\ A. Iwazaki \\ Department of Physics, Nishogakusha University, Ohi 2590, Shonan-machi, 277 Japan
}

\begin{abstract}
In certain double layer quantum Hall systems we show the existence of Josephson phenomena such as the Josephson effect, the Meissner effect and the Anderson plasmon excitation. The distinctive feature is that the unit of the charge associated with the Josephson tunneling is $e$ of the single electron and not $2 e$ of the Cooper pair. We develop a theory of these Josephson phenomena by using the Chern-Simons formulation of the planar electrons. We explain our results by using the pseudospin language. Furthermore, we point out that Anderson plasmon excitations may have already been observed by a recent experiment on a double layer quantum Hall system. A microwave experiment without attaching external leads is proposed for a direct observation of plasmon excitations.
\end{abstract}

\section{INTRODUCTION}

The two-dimensional electron system has a rich ground state structure. A most prominent discovery is the fractional quantum Hall (QH) effect in a strong magnetic field [1]. The fractional QH effect is well known and analyzed at odd-denominator Landau-level filling factors in the single layer system. On the other hand, the double layer (DL) system has recently attracted much attention. As an intralayer transport property it has revealed new QH states with even-denominator filling factors [2]. As an interlayer transport property a new phenomenon of the Josephson tunneling has been predicted in a certain QH system [3-8].

As a specific feature of the two dimensional space, the particle does not possess the definite spin-statistics relation [9], which leads to a remarkable possibility that in the presence of an external magnetic field an electron can be turned into a boson by making an electron-flux composite (bosonized electron). The QH state is considered as a condensed phase of such bosonized electrons [10]. With the ground state being a condensed phase of electrons, it is natural to anticipate the Josephson effect also in a DL system in an analogy to superconductor with the Cooper pair condensation. The Josephson effect must yield the characteristic feature of the condensation of unpaired electrons, demonstrating statistics transmutation on the plane.

The existence of Josephson behaviors in a certain double layer QH (DLQH) system is physically understood based on the Halperin wave function. At a generic filling factor $\nu=2 /(m+n)$ with $m$ and $n$ integers ( $m$ is odd), the DLQH state is described by the wave function [11]:

$$
\Psi\left(N_{1}, N_{2}\right)=e^{i \eta\left(N_{1}, N_{2}\right)}
$$

$$
\begin{aligned}
& \times \prod\left(\bar{z}_{r}^{1}-\bar{z}_{s}^{1}\right)^{m} \prod\left(\bar{z}_{p}^{2}-\bar{z}_{q}^{2}\right)^{m} \prod\left(\bar{z}_{r}^{1}-\bar{z}_{p}^{2}\right)^{n} \\
& \times \exp \left\{-\frac{1}{4} e B\left(\sum\left|z_{r}^{1}\right|^{2}+\sum\left|z_{p}^{2}\right|^{2}\right)\right\}
\end{aligned}
$$

where the complex coordinate $z_{r}^{\alpha}$ is used for the $r$-th electron position in the layer $\alpha$, and $N_{\alpha}$ denotes the number of electrons in the layer $\alpha$. It is clear that integers $m$ and $n$ describe the intralayer and interlayer correlations of electrons, respectively. A remarkable feature appears in this wave function when $m=n$ : At the specific filling factor $\nu=1 / m$ with $m=n$, one cannot tell whether two electrons belong to the same layer or the different layers in the wave function (1.1), because the intralayer and interlayer electron correlations are identical. Namely, at the same filling factor, there are many $\mathrm{QH}$ states described by the wave functions $\Psi\left(N_{0}+\Delta N, N_{0}-\Delta N\right)$, where $\Delta N$ electrons have been transferred from one layer to the other. Corresponding to the electron number $\Delta N$, the phase $\theta$ is defined as the conjugate variable. With a weak tunneling interaction included, these $\mathrm{QH}$ states are mixed among themselves, and the eigen states of the interaction are specified with $\theta$ :

$$
\Psi(\theta)=\sum_{\Delta N} e^{i \theta \Delta N} \Psi\left(N_{0}+\Delta N, N_{0}-\Delta N\right)
$$

with the energy being proportional to $\cos \theta$. Recall that the wave function of the superconductor Josephson junction [12] is given by

$$
\Psi_{\text {super }}(\theta)=\sum_{\Delta N} e^{i \theta \Delta N} \Psi_{1}\left(N_{0}+\Delta N\right) \Psi_{2}\left(N_{0}-\Delta N\right),
$$

where $\Psi_{\alpha}\left(N_{\alpha}\right)$ describes the superconducting state in the bulk $\alpha$ with the electron number $N_{\alpha}$. Because of the 
similar structure of the wave functions we are naturally led to the anticipation for the Josephson behaviors in the present DLQH system.

Indeed, when external leads are attached to the system, the time derivative of the average of the phase $\theta$ is given by the voltage $V_{\text {ext }}$ applied between the two layers,

$$
\frac{d\langle\theta\rangle}{d t}=\left\langle\frac{\partial H}{\partial \Delta N}\right\rangle=e V_{\mathrm{ext}}
$$

and the Josephson current flows as

$$
\frac{d\langle\Delta N\rangle}{d t}=-\left\langle\frac{\partial H}{\partial \theta}\right\rangle=-J_{c} \sin \langle\theta\rangle,
$$

with $J_{c}$ being the critical current. Related phenomena also follow such as the Meissner effect, the Fraunhofer pattern in the DC-current circuit with the parallel magnetic field and the Anderson plasmon excitation. All of these are familiar in the superconductor Josephson junction [12] based on the wave function (1.3), which are analogously predicted to occur in the DLQH state based on the wave function (1.2).

However, we should mention that there is a distinctive feature on the energy $\langle H\rangle$ associated with the Josephson tunneling. In the case of the superconductor it is composed of the capacitance and tunneling energies:

$$
E_{C}=e^{2} \frac{(\Delta N)^{2}}{2 C}
$$

with $C$ the capacity of the system and

$$
E_{T}=-\Delta_{\mathrm{SAS}} N_{0} \cos \theta,
$$

with $\Delta_{\mathrm{SAS}}$ the tunneling gap. In the case of our $\mathrm{QH}$ states with $m=n$ there is an additional tunneling energy:

$$
E_{\Delta}=\frac{\Delta_{\mathrm{SAS}}(\Delta N)^{2} \cos \theta}{2 N_{0}}
$$

with $V$ the volume of the surface area. Due to this term there is an incorrect argument [13] that the Josepshon effect does not occur. In this paper we make it clear that this term is harmless to the Josephson effect just as the capacitance term $E_{C}$ is harmless.

The aim of this paper is to present a microscopic formalism of the Josephson effect and its associated phenomena in DLQH systems. For this purpose we use the Chern-Simons (CS) gauge theory of the planar electrons [14], where an electron is regarded as a composite of a boson (bosonized electron) and a CS flux of the statistical field. We carry out our analysis in the mean-field approximation together with the projection of the states of electrons to the lowest Landau level (LLL). When this CS field precisely cancels the external perpendicular magnetic field $B_{\perp}$, bosonized electrons see no net magnetic field and are condensed. This is the QH state. In the single layer system, the $\mathrm{QH}$ state is always unique at a given filling factor [14]. This is also the case in the DL case in general. However, at the filling factor $\nu=1 / \mathrm{m}$ with $m=n$, it turns out that there exist many QH states,

$$
\left\langle\psi_{1}\right\rangle=\sqrt{\rho_{1}} e^{i \theta_{1}}, \quad\left\langle\psi_{2}\right\rangle=\sqrt{\rho_{2}} e^{i \theta_{2}},
$$

which have various density difference $\Delta \rho=\frac{1}{2}\left(\rho_{1}-\rho_{2}\right)$ with $\rho_{1}+\rho_{2}$ fixed [3]. Here, $\psi_{\alpha}$ is the bosonized electron field and $\rho_{\alpha}$ is the electron number density on the layer $\alpha(\alpha=1,2)$. The ground state is such that $\Delta \rho=0$ by minimizing the capacitance energy. If the capacitance and tunneling interactions are neglected, these QH states are degenerate and a Goldstone mode appears as a phase $\theta\left(=\theta_{1}-\theta_{2}\right)$ conjugate to $\Delta \rho$. With the capacitance and tunneling interactions included, the degeneracy is removed and a coherent tunneling of electrons occurs between the two layers. In our microscopic theory we may derive an effective Hamiltonian with variables $\theta$ and $\Delta \rho$, which describes the low energy collective motions of the system associated with the coherent tunneling. The Hamiltonian together with the Maxwell equations govern all of the Josephson phenomena. We are concerned about the energy scale of the tunneling which is much smaller than the average Coulomb energy between electrons.

Although we develop our formalism based on the microscopic CS theory, it is possible and convenient to summarize our results by using the pseudospin language [15]. This helps us to make an intuitive picture on the Josephson phenomena. The pseudospin operator $\mathbf{S}$ is defined by using the bosonized electron fields as

$$
\begin{aligned}
& S_{x}=\frac{i}{2} \int d^{2} x\left(\psi_{1}^{\dagger} \psi_{2}-\psi_{2}^{\dagger} \psi_{1}\right), \\
& S_{y}=\frac{1}{2} \int d^{2} x\left(\psi_{1}^{\dagger} \psi_{2}+\psi_{2}^{\dagger} \psi_{1}\right), \\
& S_{z}=\frac{1}{2} \int d^{2} x\left(\psi_{1}^{\dagger} \psi_{1}-\psi_{2}^{\dagger} \psi_{2}\right),
\end{aligned}
$$

satisfying $\left[S_{i}, S_{j}\right]=i \varepsilon_{i j k} S_{k}$. Our microscopic Hamiltonian with $m=n$ is invariant under the SU(2) pseudospin rotation when the interlayer distance vanishes $(d=0)$. However, with a nonzero interlayer distance $(d \neq 0)$ the Coulomb interaction does not commute with $S_{x}$ and $S_{y}$. Hence, only a rotational symmetry around the pseudospin $z$ axis remains as an exact symmetry. This rotational symmetry is broken spontaneously in the $\mathrm{QH}$ states (1.9). Thus, the system resembles the XY model, where the ground state is a ferromagnet with a spontaneous magnetization $\langle\mathbf{S}\rangle \neq 0$ in the pseudospin $x y$ plane. Its direction is specified by the angle $\theta$. From (1.10c) the genierator of a rotation around the pseudospin $z$ axis is given by $S_{z}=\Delta N$. It is obvious that this angle $\theta$ is equal to the phase $\theta$ conjugate to $\Delta N$. In this way the low energy collective motions of the $\mathrm{QH}$ states correspond to the motions of the magnetization.

We see that the tunneling interaction,

$$
H_{T}=-\frac{\Delta_{\mathrm{SAS}}}{2} \int d^{2} x\left(\psi_{1}^{\dagger} \psi_{2}+\psi_{2}^{\dagger} \psi_{1}\right)=h S_{y},
$$


with $h=\Delta_{\mathrm{SAS}}$, is equivalent to applying the pseudomagnetic field with strength $h$ in the $y$ direction. Thus, its effect is to align the magnetization with the $y$ direction $(\theta=0)$.

Our effective Hamiltonian describing the low energy collective motions of the $\mathrm{QH}$ states is found to be

$$
H=e^{2} \frac{(\Delta N)^{2}}{2 C}-\Delta_{\mathrm{SAS}} N_{0} \cos \theta+\frac{\Delta_{\mathrm{SAS}}(\Delta N)^{2} \cos \theta}{2 N_{0}},
$$

for $\langle\Delta N\rangle \ll N_{0}$ together with the commutation relation

$$
[\theta, \Delta N]=i
$$

The classical limit of this Hamiltonian yields obviously the energy $E=E_{C}+E_{T}+E_{\Delta}$. In the terminology of the pseudospin it is rewritten as

$$
H=\frac{e^{2} S_{z}^{2}}{2 C}-h S_{y}^{0}+\frac{h S_{z}^{2} S_{y}^{0}}{2 N_{0}^{2}},
$$

where $\mathbf{S}^{0}=\left(S_{x}^{0}, S_{y}^{0}\right)$ is the pseudospin of the XY model:

$$
S_{x}^{0}=N_{0} \sin \theta, \quad S_{y}^{0}=N_{0} \cos \theta .
$$

Note that we have used the expansion,

$$
S_{y}=S_{y}^{0}-\frac{S_{z}^{2} S_{y}^{0}}{2\left(S^{0}\right)^{2}}
$$

with $\left(S^{0}\right)^{2}=N_{0}^{2}$ in the tunneling term (1.11) to derive (1.14), which is allowed for $\left\langle S_{z}\right\rangle \ll N_{0}$.

It follows from the Hamiltonian that, when there is no pseudo-magnetic field $(h=0)$, the eigen states are specified by the $z$ component of the pseudospin $S_{z}(=\Delta N)$ with the wave functions $\Psi\left(N_{0}+\Delta N, N_{0}-\Delta N\right)$. These states have only the capacitance energy $e^{2}(\Delta N)^{2} / 2 C$, and they are almost degenerate when the capacity $C$ is very large (as it is). They may be reorganized to give the eigen states of the pseudospin angle $\theta$, as is the wave function $\Psi(\theta)$ in (1.2).

Once the pseudo-magnetic field is applied $(h \neq 0)$, the pseudospin is aligned so that $\theta=0$. Quantum mechanically the pseudospin direction oscillates around the magnetization $\left\langle\mathbf{S}^{0}\right\rangle$, in $\theta$ as well as in $S_{z}$, with frequency $\omega_{P}$ :

$$
\omega_{P}=\sqrt{\omega_{J}^{2}+\Delta_{\mathrm{SAS}}^{2}},
$$

where $\omega_{J}=e^{2} N_{0} \Delta_{\mathrm{SAS}} / C$. This oscillation mode is called the Anderson plasmon in superconductor Josephson junction [12]. It is named as plasmon since the mode is accompanied by the oscillation of the charging $(e \Delta N)$ between the two layers. In the formula for $\omega_{P}$, the second term in the square root comes from the third term in the Hamiltonian which gives the energy $E_{\Delta}$. Thus, this term simply changes the frequency of the Anderson plasmon and is harmless for the Josephson phenomena.
As we shall see, this change of the plasmon frequency can be observed experimentally in an interesting way.

When external leads are attached to each layers, the system shows an entirely different aspect because there exists no charging $(\Delta N=0)$ on the layers. Because of this fact the oscillation mode of the pseudospin becomes gapless (or the frequency vanishes): There is no force acting on the pseudospin to pull it back to the $y$ axis. When an external voltage $V_{\text {ext }}$ is applied between the two layers, the pseudospin rotates around the $z$ axis with frequency $e V_{\text {ext }}$. Indeed, the Hamiltonian now reads

$$
H_{V}=H+e V_{\text {ext }} S_{z}
$$

which govern the dynamics of the pseudospin. The equations of motion are

$$
\begin{aligned}
& \frac{d \theta}{d t}=i\left[H_{V}, \theta\right]=e V_{\mathrm{ext}}, \\
& \frac{d S_{z}}{d t}=i\left[H_{V}, S_{z}\right]=-J_{c} \sin \theta \propto S_{x},
\end{aligned}
$$

with $J_{c}=\Delta_{\mathrm{SAS}} N_{0}$, where we have taken into account that there exists no charging $\left(S_{z}=\Delta N=0\right)$. Physically, $d\left\langle S_{z}\right\rangle / d t$ is the Josephson current $d\langle\Delta N\rangle / d t$ flowing between the layers, and according to these equations the AC Josephson effect with DC-voltage feed occurs. Similarly, the Josephson effect with the DC-current feed is possible.

Rich physics is expected when we apply the parallel magnetic field $B_{\|}$between the two layers. It is easy to see that the effect of the uniform parallel field $B_{k}$ inside the junction is to change locally the phase difference $\theta$ between the electron condensations (1.9) in each layers:

$$
\partial_{x} \theta=-e d B_{y} .
$$

Here, we have applied the parallel magnetic field in the $y$ direction. Note that $B_{y}$ is in general different from the external field $B_{\|}$due to the screened effect. Thus, the magnetization $\left\langle\mathbf{S}^{0}\right\rangle$ modulates smoothly its direction along the $x$ axis by the penetrated magnetic field $B_{y}$ between the two layers.

Because of this local modulation of the magnetization with angle $\theta$, the tunneling current $J_{c}\langle\sin \theta\rangle$ takes a nonvanishing value even in an isolated system. This leads to the Meissner effect due to this Josephson current just as in superconductor Josephson junction. In order to discuss the effect we have to address the Maxwell equations as well as the equations of motion for $\theta$ and $\Delta p$. By solving them, we obtain the conclusion [4] that the external magnetic field $B_{\|}$is completely screened with the penetration depth $\sim 0.03 \mathrm{~mm}$ as far as $B_{\|}$is less than 100Gauss approximately for a typical sample with $\rho=10^{11} / \mathrm{cm}^{2}, \Delta_{\mathrm{SAS}}=1 \mathrm{~K}$ and $d=100 \AA$. Namely, as a result of the screening, it follows that $B_{y}=0$ and $\theta=0$ in (1.20) except for the vicinity of the edge of the layer, where the parallel magnetic field penetrates. The existence of the Meissner effect is not accidental. It is ensured 
by the spontaneous symmetry breakdown of the electromagnetic gauge symmetry. The symmetry breakdown yields a mass to the photon, which gives the penetration depth. The mass is just the plasmon frequency $\omega_{P}$.

It is important to analyze how the plasmon frequency $\omega_{P}$ is modified in a sufficiently strong external magnetic field $B_{\|}$. In such a case the screening effect becomes insufficient and the external parallel magnetic field penetrates into the junction freely. From (1.20) we find that

$$
\langle\theta\rangle=\theta_{0}-x e d B_{\|}
$$

with $\theta_{0}$ a constant. The pseudospin oscillates around the axis of the magnetization $\left\langle\mathbf{S}^{0}\right\rangle$ which is modulated by the angle (1.21). This oscillation mode is observable, and there is an intriguing possibility that it has already been observed in a recent experiment [16].

A recent experiment on a DLQH system [16] has revealed an anomalous behavior in the activation energy versus the parallel magnetic field. It is to be stressed that this anomaly has only been observed in the system where the Josephson effect had been predicted. There is an attempt [13] to understand this anomaly as a commensurate-incommensurate phase transition, but this attempt is not satisfactory. In this paper we explain the anomaly by using Anderson plasmons [6].

Anderson plasmons are neutral, but they are oscillation modes of the charging $e \Delta N$ in each layer, $\Delta N \propto$ $\sin \left(\omega_{P} t\right)$. Therefore, plasmon excitations affect transport phenomena. In particular, we expect that the activation energy $E^{A}$ determined by measuring the electric resistivity $\rho_{x x}$ should detect their excitations. We have called the pseudospin oscillation around the magnetization $\left\langle\mathbf{S}^{0}\right\rangle$ the plasmon mode. The plasmon frequency is a function of the parallel magnetic field $B_{\|}$or the tilt angle $\Theta\left(\tan \Theta=B_{\|} / B_{\perp}\right)$. The activation energy is then found to be

$$
E^{A}(\Theta)=\sqrt{\omega_{J}^{2}\left|\frac{\sin \left(\pi \tan \Theta / \tan \Theta_{c}\right)}{\pi \tan \Theta / \tan \Theta_{c}}\right|+\Delta_{\mathrm{SAS}}^{2}}
$$

for $\Theta<\Theta_{c}$ and

$$
E^{A}(\Theta)=\Delta_{\mathrm{SAS}},
$$

for $\Theta>\Theta_{c}$ with a critical angle $\Theta_{c}$. The formula contains three parameters $\omega_{\mathcal{J}}, \Delta_{\mathrm{SAS}}$ and $\Theta_{\boldsymbol{c}}$. With an appropriate choice of them, we can account for the anomalous behavior in the activation energy [16] quite well; see figures in section 5 . In this paper we use the units such that $c=1$ and $\hbar=1$.

This paper is composed as follows. In section 2, from a physical point of view, we explain why unpaired planar electrons with Coulomb repulsion may be turned into bosons in the presence of an appropriate external magnetic field. In section 3 we analyze the DLQH system together with the LLL projection. In the case of the filling factor $\nu=1 / m$ with $m=n$, we derive the effective
Hamiltonian describing the low energy collective motion of the system in terms of $\theta$ and $\Delta \rho$. In section 4 , using the Hamiltonian we show the existence of the Josephson effect, the Meissner effect and other Josephson phenomena. We examine in detail how the QH-state Josephson behaviors are similar to and different from the superconductor Josephson behaviors. In section 5 we explain the activation energy anomaly found in recent experiments [16] by using our plasmon excitations. We also propose a microwave experiment for a direct observation of plasmon excitations.

\section{SINGLE LAYER QUANTUM HALL SYSTEMS}

It is a specific feature of the two dimensional space that the particle does not possess the definite spin-statistics relation and hence that an electron can be turned into a boson by making a composite with flux. This leads to a remarkable possibility that unpaired electrons can be condensed in appropriate circumstances. Let us review a physical picture how such a condensation of unpaired electrons is possible on a plane in the presence of an external magnetic field [5].

It is essential that electrons, making cyclotron motions, are uniformly distributed on the plane due to the Coulomb repulsion. It is reasonable to simulate the system by placing electrons on lattice points with equal spacing and with all lattice points occupied. Due to the Coulomb interaction electrons exchange their positions. Thus, the electrons move around all the lattice points. In this lattice approximation the external magnetic field is also distributed among the lattice points: to each lattice point the magnetic flux $B / \rho$ is attached, with $\rho$ being the electron density. Then, when two electrons exchange their positions, the wave function reads as $\Psi(y, x)=-e^{i \alpha} \Psi(x, y)$ with $\alpha=e B / 2 \rho$ by acquiring the Aharanov-Bohm phase $e^{i \alpha}[17]$. When the magnetic field $B$ is such that $\alpha=\pi m$ with $m$ being an odd integer, $\Psi(x, y)$ becomes a wave function of bosons, which are nothing but bosonized electrons. Then, these bosonized electrons would make a condensed phase, the $\mathrm{QH}$ fluid, at the magic filling factor $\nu \equiv 2 \pi \rho / e B=1 / m$.

A field-theoretical realization of this picture of the $\mathrm{QH}$ state is given by the CS gauge theory, as we now explain, where the electron is described by the bosonized electron together with the CS gauge field representing the statistics of the electron. Namely, the electron is regarded as a composite of a boson and a CS gauge flux. In the presence of a repulsive Coulomb interaction, the mean-field ground state at the magic filling factor is such that the CS flux is cancelled out by the external magnetic flux, and hence the bosonized electrons, thus identified as composites of electrons and magnetic flux, make a condensation.

Bosonized electrons are defined trivially in terms of the wave functions. Let $\widehat{\Psi}\left(x_{1}, \cdots, x_{N}\right)$ be the wave function 
of $N$ electrons on a plane. Then, the wave function of the bosonized electrons $\Psi\left(x_{1}, \cdots, x_{N}\right)$ is defined by

$$
\Psi\left(x_{1}, \cdots, x_{N}\right) \equiv e^{i m \sum_{r<\cdot} \theta\left(x_{r}-x_{\theta}\right)} \widehat{\Psi}\left(x_{1}, \cdots, x_{N}\right),
$$

where $\theta\left(x_{r}-x_{s}\right)$ is the azimuthal angle between two electrons located at $x_{r}$ and $x_{s}$. We call an odd integer $m$ the statistics parameter. By exchanging any two electrons, $\Psi$ transforms obviously as a bosonic wave function. (The bosonic wave function satisfies the hard-core condition, $\Psi=0$ for $x_{r}=x_{s}$.) The procedure (2.1) is known as a singular gauge transformation. By this transformation the momentum operator of the bosonized electron reads

$$
i \partial_{k}^{r}+a_{k}\left(\dot{x_{r}}\right),
$$

with $a_{k}\left(x_{r}\right)=m \sum_{s \neq r} \partial_{k}^{r} \theta\left(x_{r}-x_{s}\right)$ or

$$
\varepsilon_{i j} \partial_{i} a_{j}(x)=2 \pi m \sum_{s} \delta\left(x-x_{s}\right) .
$$

In this way the bosonization is simply a procedure to take off an appropriate phase factor.

The second quantization is also trivially made, which leads to a CS gauge theory. After second quantization, (2.3) yields

$$
\varepsilon_{i j} \partial_{i} a_{j}=2 \pi m \psi^{\dagger} \psi
$$

which defines the statistical gauge field $a_{k}$ in terms of the bosonized electron field $\psi$, satisfying

$$
\left[\psi(x), \psi^{\dagger}(y)\right]=\delta(x-y) .
$$

The bosonized electron is a hard-core boson. The statistical gauge field $a_{k}$, representing solely the phase degree of freedom, has no independent dynamics. The field $a_{k}$ is also called the CS gauge field.

The Hamiltonian is simply given by

$$
\mathcal{H}=\frac{1}{2 M} \int d^{2} x\left|D_{k} \psi\right|^{2}+\mathcal{V}[\psi],
$$

with

$$
i D_{k}=i \partial_{k}+a_{k}-e A_{k}
$$

in terms of the bosonized electron field $\psi$ and the CS gauge field $a_{k}$ subject to (2.4); $M$ is the effective mass of electrons; $N$ is the total number of electrons. We have taken the layer parallel to the $x y$ plane. The external magnetic field $B_{\perp}$ is applied in the $z$ direction perpendicular to the layer:

$$
A_{k}=-\frac{1}{2} B_{\perp} \varepsilon_{k j} x^{j} .
$$

Term $\mathcal{V}[\psi]$ represents the intralayer Coulomb interactions that drive the planar electron system into the QH liquid:

$$
\begin{aligned}
\mathcal{V}[\psi]= & \frac{e^{2}}{2 \varepsilon} \int d^{2} x d^{2} y:\left\{\psi^{\dagger} \psi(x)-\rho_{0}\right\} \\
& \times \frac{1}{|x-y|}\left\{\psi^{\dagger} \psi(y)-\rho_{0}\right\}:
\end{aligned}
$$

where $\rho_{0}$ stands for the constant neutralizing background charge and $\varepsilon$ for the dielectric constant. In these expressions the ordering of the operator $|\mathcal{O}|^{2}$ is $\mathcal{O}^{\dagger} \mathcal{O}$, and the dots : $\mathcal{O}$ : denote the normal ordering of the operator $\mathcal{O}$. With these operator orderings it can be proved [18] that the Hamiltonian (2.6) leads to exactly the $N$ body Schrödinger equation of motion of electrons.

It is convenient to rewrite the Hamiltonian by using the Bogomol'nyi decomposition [19]:

$$
\begin{aligned}
\int d^{2} x\left|D_{k} \psi\right|^{2}= & \int d^{2} x\left(\left|\left(D_{1}-i D_{2}\right) \psi\right|^{2}\right. \\
& \left.+M \omega_{c}:|\psi|^{2}:-2 m \pi:|\psi|^{4}:\right),
\end{aligned}
$$

where $\omega_{c}=e B / M$ is the cyclotron frequency. Here, the last term represents the contact interaction, which can be discarded for the hard-core bosons. Hence, we obtain [14]

$$
\mathcal{H}=\frac{1}{2 M} \int d^{2} x\left|\left(D_{1}-i D_{2}\right) \psi\right|^{2}+\frac{1}{2} \omega_{c} N+\mathcal{V}[\psi],
$$

which defines a microscopic field theory of the planar system of $\mathrm{N}$ electrons. This Hamiltonian is invariant under the global phase transformation:

$$
\psi \rightarrow e^{i \Lambda} \psi,
$$

with a constant $\Lambda$. This symmetry is associated with the conservation of the electron number.

If the Coulomb interaction is switched off the Hamiltonian (2.11) simply describes cyclotron motions of electrons. When all $N$ electrons occupy the LLL, the total kinetic energy is $\frac{1}{2} \omega_{c} N$. In this case it is obvious from this Hamiltonian that the states $|f\rangle$ satisfying

$$
\left(D_{1}-i D_{2}\right) \psi|f\rangle=0
$$

have the energy $\frac{1}{2} \omega_{c} N$ of the LLL. We call this equation the LLL constraint.

We prove that the LLL constraint (2.13) defines surely the states in the LLL. For this purpose we analyze the wave function

$$
\Psi_{f} \equiv\left\langle 0\left|\psi\left(x_{1}\right) \psi\left(x_{2}\right) \cdots \psi\left(x_{N}\right)\right| f\right\rangle,
$$

with $\psi(x)|0\rangle=0$. Introducing the complex coordinate $z=x+i y$ for each electron, we rewrite the LLL constraint (2.13) as

$$
\left(\frac{\partial}{\partial z_{r}}+\frac{e B}{4} \bar{z}_{r}-\frac{m}{2} \sum_{s \neq r} \frac{1}{z_{r}-z_{s}}\right) \Psi_{f}=0 .
$$

This equation can be exactly solved $[14,20]$. By recovering the phase factor given by (2.1) the solution is given by 


$$
\widehat{\Psi}_{f}=F(\bar{z}) \prod\left(\bar{z}_{r}-\bar{z}_{s}\right)^{m} \exp \left\{-\frac{1}{4} e B \sum\left|z_{r}\right|^{2}\right\},
$$

where $F(\bar{z})$ is an arbitrary analytic function. The arbitrariness in $F(\bar{z})$ implies the degeneracy of the states in the LLL. As is well known, this is the most general form of the wave function of the electrons in the LLL. Hence, the condition (2.13) selects the LLL states in the second quantized formalism of bosonized electrons.

The real ground state is determined by minimizing the Coulomb energy (2.9) in the LLL states. Namely, we diagonalize the Coulomb interaction $\mathcal{V}$, i.e., the matrix $\langle g|\mathcal{V}| f\rangle$ with $|f\rangle$ and $|g\rangle$ being LLL states obeying (2.13). An exact treatment is practically impossible. Our strategy is to use the semiclassical quantization, in which we first determine the mean-field ground state and then to take into account Gaussian fluctuations around it.

In the mean-field approximation, the LLL states are given by solving the classical equation

$$
\left(D_{1}-i D_{2}\right) \psi=0
$$

which is found [14] to contain ensembles of vortices in general, implying the degeneracy of the LLL states. One vortex carries a Coulomb energy of the order of $e^{2} / \ell_{B}$ with $\ell_{B} \equiv 1 / \sqrt{e B}$ being the magnetic radius. Among these solutions the Coulomb energy (2.9) is minimized by the solution, $a_{k}=e A_{k}$ and $\psi=\sqrt{\rho} e^{i \theta}$, with constant phase $\theta$ and density $\rho$ if it is a possible one. Then, the mean-field ground state is given by

$$
\left\langle a_{k}\right\rangle=e A_{k}, \quad\langle\psi\rangle=\sqrt{\rho} e^{i \theta},
$$

as corresponds to a uniform distribution of electrons. It is realized only at the magic filling factor $\nu=1 / m$ due to the constraint equation (2.4).

This mean-field ground state illustrates our physical picture of the condensation of bosonized electrons, as we have promised. Although the decomposition of an electron into a bosonized electron and a CS flux is just a mathematical trick, the CS flux is traded with the external magnetic flux in this mean-field ground state. Then, the bosonized electrons acquire a physical reality as composites of electrons and magnetic flux. They are condensed in the zero-momentum state; the QH state is a condensed phase of bosonized electrons. Note that this condensation occurs only at the magic filling factor; in its vicinity the ground states contain ensembles of topological vortices (quasiparticles) which are excited but trapped by impurities on this condensate. This leads to the formation of plateau in the Hall conductance around the magic filling factor.

It is important to note that the QH state (2.18) breaks spontaneously the phase symmetry (2.12). Thus, it seems to generate a Goldstone mode. However, actually no Goldstone mode is generated in this CS gauge theory because the constraint equation (2.4) leads to nonlocal CS interactions. Indeed, it can be solved as

$$
a_{i}(x)=\frac{\alpha}{\pi} \int d^{2} y \frac{\varepsilon_{i j}(x-y)_{j}}{|x-y|^{2}}|\psi(y)|^{2} .
$$

As is well known, the Goldstone theorem does not hold in the presence of the nonlocal interaction.

It is straightforward to carry out the analysis of the Gaussian fluctuations around the mean-field ground state (2.18). In so doing we can verify that the ground state is stable and that it is described by the Laughlin wave function. However, since this is not the main topics of this paper, we only cite references [14].

\section{DOUBLE LAYER QUANTUM HALL SYSTEMS}

The CS gauge theory of the DL electron system is similarly constructed. The wave function of the bosonized electrons $\Psi$ is defined by taking off the phase factors from that of the electrons $\widehat{\Psi}$ as in (2.1):

$$
\Psi \equiv e^{i m \sum_{r<\rho} \theta\left(x_{r}^{1}-x_{\bullet}^{1}\right)} e^{i m \sum_{p<q} \theta\left(x_{p}^{2}-x_{q}^{2}\right)} e^{i n \sum \theta\left(x_{r}^{1}-x_{p}^{2}\right)} \widehat{\Psi},
$$

with the statistics parameters $m$ and $n$; here, $m$ is an odd integer but $n$ is any integer. The system exhibits entirely different properties depending on the statistics parameters being $m=n$ or not. First we briefly review the case with $m \neq n$.

\section{A. Systems with $m \neq n$}

It is clear from (3.1) that, when $m \neq n$, two CS fields are necessary to extract the phase degrees of freedom and to bosonize electrons. In second-quantized theory, these two CS gauge fields $a_{k}^{\alpha}$ are defined by

$$
\begin{aligned}
& \varepsilon_{i j} \partial_{i} a_{j}^{1}=2 \pi\left(m \psi_{1}^{\dagger} \psi_{1}+n \psi_{2}^{\dagger} \psi_{2}\right), \\
& \varepsilon_{i j} \partial_{i} a_{j}^{2}=2 \pi\left(n \psi_{1}^{\dagger} \psi_{1}+m \psi_{2}^{\dagger} \psi_{2}\right),
\end{aligned}
$$

in terms of the bosonized electron fields $\psi_{\alpha}$, where $\alpha$ is the layer index: $\alpha=1,2$.

The Hamiltonian is given by [3]

$$
\mathcal{H}=\sum_{\alpha} \frac{1}{2 M} \int d^{2} x\left|\left(D_{1}^{\alpha}-i D_{2}^{\alpha}\right) \psi_{\alpha}\right|^{2}+\frac{1}{2} \omega_{c} N+\mathcal{V}[\psi]
$$

where

$$
i D_{k}^{\alpha}=i \partial_{k}+a_{k}^{\alpha}-e A_{k},
$$

with (2.8). The Coulomb term $\mathcal{V}$ is given by

$$
\mathcal{V}[\psi]=\mathcal{V}_{11}[\psi]+\mathcal{V}_{22}[\psi]+\mathcal{V}_{12}[\psi]
$$

with 


$$
\begin{aligned}
\mathcal{V}_{\alpha \beta}[\psi]= & \frac{e^{2}}{2 \varepsilon} C_{\alpha \beta} \int d^{2} x d^{2} y:\left\{\psi_{\alpha}^{\dagger} \psi_{\alpha}(x)-\rho_{0}\right\} \\
& \times \frac{1}{\sqrt{(x-y)^{2}+d_{\alpha \beta}^{2}}}\left\{\psi_{\beta}^{\dagger} \psi_{\beta}(y)-\rho_{0}\right\}:
\end{aligned}
$$

where $d_{11}=d_{22}=0$ and $d_{12}=d$ is the interlayer distance; $C_{11}=C_{22}=1$ and $C_{12}=2 ; e \rho_{0}$ is the background charge in each layer.

The LLL constraint reads

$$
\left(D_{1}^{\alpha}-i D_{2}^{\alpha}\right) \psi_{\alpha}|f\rangle=0
$$

which is exactly solvable: After recovering the phase factor as given by (3.1) the wave function is found to be [3]

$$
\begin{aligned}
F\left(\bar{z}_{1}, \bar{z}_{2}\right) & \prod\left(\bar{z}_{r}^{1}-\bar{z}_{s}^{1}\right)^{m} \prod\left(\bar{z}_{p}^{2}-\bar{z}_{q}^{2}\right)^{m} \prod\left(\bar{z}_{r}^{1}-\bar{z}_{p}^{2}\right)^{n} \\
& \times \exp \left\{-\frac{1}{4} e B\left(\sum\left|z_{r}^{1}\right|^{2}+\sum\left|z_{p}^{2}\right|^{2}\right)\right\}
\end{aligned}
$$

with an arbitrary analytic function $F\left(\bar{z}_{1}, \bar{z}_{2}\right)$. This arbitrariness is fixed in the real ground state by minimizing the Coulomb energy (3.5).

The mean-field ground state representing the QH state is given by

$$
\left\langle a_{k}^{\alpha}\right\rangle=e A_{k}, \quad\left\langle\psi_{\alpha}\right\rangle=\sqrt{\rho_{0}} e^{i \theta_{\alpha}},
$$

with constant phase $\theta_{\alpha}$ and constant density $\rho_{0}$. It should be stressed that, due to the constraint equations (3.2), the electron density is fixed to be equal on the two layers as in (3.9) and that this state is realized only at the magic filling factor

$$
\nu \equiv \frac{2 \pi \rho}{e B}=\frac{2}{m+n},
$$

with $\rho=2 \rho_{0}$. For instance, the $\mathrm{QH}$ state experimentally observed [2] at $\nu=\frac{1}{2}$ is explained by choosing $m=3$ and $n=1$. The DLQH state is shown [3] to be incompressible when $m \neq n$.

We remark the following symmetry property. The Hamiltonian (3.3) possesses two global phase symmetries:

$$
\psi_{1}(x) \rightarrow e^{i \Lambda} \psi_{1}(x), \quad \psi_{2}(x) \rightarrow e^{-i \Lambda} \psi_{2}(x),
$$

and

$$
\psi_{1}(x) \rightarrow e^{i \Lambda} \psi_{1}(x), \quad \psi_{2}(x) \rightarrow e^{i \Lambda} \psi_{2}(x) .
$$

Although these two symmetries are broken spontaneously, no Goldstone modes are generated because the conserved charges associated with these two symmetries couple with the CS gauge fields $a_{k}^{\alpha}$ and because these fields $a_{k}^{\alpha}$ generate nonlocal CS interactions by the constraint equations (3.2). The Goldstone theorem does not hold because of nonlocal interactions also in this case.

Analyzing the Gaussian fluctuation around the meanfield ground state, we can verify [3] that the ground state is described by the Halperin wave function [11], i.e., by (3.8) with $F=1$, or

$$
\begin{aligned}
\prod\left(\bar{z}_{r}^{1}-\right. & \left.\bar{z}_{s}^{1}\right)^{m} \prod\left(\bar{z}_{p}^{2}-\bar{z}_{q}^{2}\right)^{m} \prod\left(\bar{z}_{r}^{1}-\bar{z}_{p}^{2}\right)^{n} \\
& \times \exp \left\{-\frac{1}{4} e B\left(\sum\left|z_{r}^{1}\right|^{2}+\sum\left|z_{p}^{2}\right|^{2}\right)\right\} .
\end{aligned}
$$

The role of the statistics parameters $m$ and $n$ is manifest in this wave function: $m$ describes the intralayer correlation, while $n$ the interlayer correlation. These correlations are basically generated by the intralayer and interlayer Coulomb interactions. Thus, to realize the state characterized by $m$ and $n$ it is necessary to choose the interlayer distance $d$ appropriately: Namely, the interlayer correlation parameter $n$ depends on the interlayer distance $d$. In particular, when $m=n$, the electrons have the same intralayer and interlayer correlations as is clear in (3.13). Hence, in order to realize such a QH state it is necessary to choose the interlayer distance $d$ to be the order of the magnetic radius $\ell_{B}$.

\section{B. Systems with $m=n$}

In this subsection we analyze the case $m=n$ in detail. Physics is very different in this case from the one with $m \neq n$. As we have described in the Introduction, at the specific filling factor $\nu=1 / m$ with $m=n$, there are many $\mathrm{QH}$ states described by the Halperin wave functions $\Psi\left(N_{0}+\Delta N, N_{0}-\Delta N\right)$ with (1.1).

This fact can be understood in our CS formalism as follows. The crucial point is that the constraint equations (3.2) are reduced to a single equation:

$$
\varepsilon_{i j} \partial_{i} a_{j}=2 \pi m\left(\psi_{1}^{\dagger} \psi_{1}+\psi_{2}^{\dagger} \psi_{2}\right)
$$

where $a_{j}=a_{j}^{1}=a_{j}^{2}$ : Namely, the system contains only one CS gauge field. As we shall discuss more in detail later, this constraint restricts only the total electron density $\rho=\rho_{1}+\rho_{2}$ in the QH states with $\rho=e B / 2 \pi m$, $\left\langle\psi_{\alpha}\right\rangle=\sqrt{\rho_{\alpha}} e^{i \theta_{\alpha}}$, and does not restrict the difference $\rho_{1}-\rho_{2}$. Therefore, there exists many $\mathrm{QH}$ states, which are degenerate in the density difference $\rho_{1}-\rho_{2}$ if the capacitance energy is ignored. Because of this fact there appears a Goldstone mode [3,5], which is identified with a gapless mode known in literature $[15,21]$.

In a clear contrast to the case with $m \neq n$, this Goldstone mode is associated with the global phase symmetry (3.11). In the present case, although two symmetries (3.11) and (3.12) are broken spontaneously in the QH states as before, there exists only one constraint equation that leads to nonlocal CS interactions. It is easy to see that only the conserved charge associated with (3.12) couples with the CS field $a_{k}$ : The generator of the symmetry (3.11), which is the electron-number difference between the two layers,

$$
\Delta N=\frac{1}{2} \int d^{2} x\left(\psi_{1}^{\dagger} \psi_{1}-\psi_{2}^{\dagger} \psi_{2}\right)
$$


does not couple with the CS field $a_{k}$. Hence, the Goldstone theorem holds that guarantees the existence of the Goldstone mode associated with the symmetry (3.11). When the tunneling is allowed between the two layers, this mode leads to a coherent tunneling of electrons, as induces various Josephson phenomena.

It may be instructive to interpret various concepts by using the pseudospin language $[15,13]$, where "up" and "down" pseudospins are assigned to electrons belonging to the first and second layers, respectively. The $z$ component $\left(S_{z}\right)$ of the total spin $(\mathbf{S})$ is just equal to $\Delta N$. Therefore, the operator $\Delta N\left(=S_{z}\right)$ is the generator of a rotation around the $z$ axis in the pseudospin space. Similarly, the generators $S_{x}$ and $S_{y}$ are defined by

$$
\begin{aligned}
& S_{x}=\frac{i}{2} \int d^{2} x\left(\psi_{1}^{\dagger} \psi_{2}-\psi_{2}^{\dagger} \psi_{1}\right), \\
& S_{y}=\frac{1}{2} \int d^{2} x\left(\psi_{1}^{\dagger} \psi_{2}+\psi_{2}^{\dagger} \psi_{1}\right), \\
& S_{z}=\frac{1}{2} \int d^{2} x\left(\psi_{1}^{\dagger} \psi_{1}-\psi_{2}^{\dagger} \psi_{2}\right),
\end{aligned}
$$

satisfying $\left[S_{i}, S_{j}\right]=i \varepsilon_{i j k} S_{k}$. With the zero interlayer spacing $(d=0)$ the system possesses the full SU(2) pseudospin symmetry. Namely, the system has the same energy when the pseudospin may point any direction in the 3 dimensional pseudospace. However, with a nonzero interlayer spacing $(d \neq 0)$, the system possesses only the pseudospin rotational symmetry generated by $S_{z}$, because the Coulomb term (3.6) does not cummute with $S_{x}$ and $S_{y}$. As we have stated above, this symmetry is spontaneously broken in the QH states, or the direction of the pseudospin is spontaneously chosen in the ground state, as is the ferromagnet. The ferromagnet states correspond to the states $\Psi(\theta)$ in (1.2), and are degenerate in the pseudospin direction $\theta$. Hereafter, we may frequently refrase our results on the Josephson tunneling in terms of the pseudospin language.

In order to analyze the coherent tunneling and associated Josephson phenomena we switch on the electromagnetic gauge interactions and the tunneling interaction. Then, the microscopic Hamiltonian density of the DL electron system is given by $[5,6]$

$$
\begin{aligned}
\mathcal{H}= & \sum_{\alpha}\left(\frac{1}{2 M}\left|\left(D_{x}^{\alpha}-i D_{y}^{\alpha}\right) \psi_{\alpha}\right|^{2}+e A_{0}^{\alpha}\left|\psi_{\alpha}\right|^{2}\right)+\frac{1}{2} \omega_{c} N \\
& -\lambda\left(\psi_{1}^{\dagger} e^{-i e d A_{z}} \psi_{2}+\psi_{2}^{\dagger} e^{i e d A_{z}} \psi_{1}\right)
\end{aligned}
$$

with

$$
i D_{k}^{\alpha}=i \partial_{k}+a_{k}-e A_{k}^{\alpha} \text {. }
$$

Here, the CS field $a_{k}$ is defined by (3.14), while $A_{\mu}^{\alpha}$ is the electromagnetic potential at the layer $\alpha$ with $\mu$ taking a value on $(0, x, y)$. We may write $A_{k}^{\alpha}=A_{k}^{\prime \alpha}+A_{k}$, where $A_{k}$ describes the perpendicular magnetic field $B_{\perp}$ as in (2.8). The additional field ${A^{\prime}}_{k}^{\alpha}$ describes weak parallel components of the external magnetic field and the field
$A_{0}^{\alpha}$ the voltage between the two layers, both of which are relevant to Josephson phenomena. In this Hamiltonian we have not included the static Coulomb interaction term between the electrons. The Coulomb interaction is crucial to drive the system into $\mathrm{QH}$ states, but once a QH state is chosen, its relevance to Josephson phenomena is by way of the electric capacity of the double layers, but this effect is taken into account through the Maxwell equations; see (3.64) below.

We give a few comments on the tunneling term in (3.17), which reads

$$
\mathcal{H}_{T}=-\lambda\left(\psi_{1}^{\dagger} \psi_{2}+\psi_{2}^{\dagger} \psi_{1}\right)
$$

in the absence of the electromagnetic fields. The first comment is on the single-particle (SP) gap energy. By way of the SP symmetric and antisymmetric field operators defined by

$$
\psi_{S}=\frac{1}{\sqrt{2}}\left(\psi_{1}+\psi_{2}\right), \quad \psi_{A S}=\frac{1}{\sqrt{2}}\left(\psi_{1}-\psi_{2}\right)
$$

the tunneling Hamiltonian $\mathcal{H}_{T}$ is diagonalized as

$$
\mathcal{H}_{T}=-\lambda\left(\left|\psi_{S}\right|^{2}-\left|\psi_{A S}\right|^{2}\right),
$$

which implies an SP energy gap

$$
\Delta_{\mathrm{SAS}}=2 \lambda,
$$

between the SP symmetric $\left(\psi_{S}\right)$ and antisymmetric $\left(\psi_{A S}\right)$ states. These states turn out to be the so-called symmetric and antisymmetric states in a double well potential problem. When the tunneling energy $\left(\Delta_{\mathrm{SAS}}\right)$ is larger than the Coulomb energies $\left(e^{2} / \varepsilon \ell_{B}\right), \mathrm{QH}$ states are realized on the symmetric state or effectively as single-layer $\mathrm{QH}$ states. In this paper we assume that $\Delta_{\mathrm{SAS}} \ll e^{2} / \varepsilon \ell_{B}$, and hence we can regard the system as genuine DLQH states.

In the pseudospin language the tunneling interaction (3.19) is rewritten as

$$
H_{T}=-2 \lambda S_{y}=-\mathrm{h} \cdot \mathrm{S},
$$

with the pseudospin generator (3.16b), which may be interpreted that an external pseudo-magnetic field $h$ is applied in the $y$ direction with $h=2 \lambda$. Then, this term breaks explicitly the pseudospin rotational symmetry associated with $S_{z}$, and enforces the direction of the magnetization to align with the $y$ axis. The strength $h$ of the symmetry breaking is assumed to be much smaller than the Coulomb energy.

The next comment is on the gauge invariance of the system. The tunneling term (3.19) breaks explicitly the phase symmetry (3.11), or the pseudospin rotation around the $z$ axis. So, it seems that the Goldstone mode is killed by this term. This is not correct since the phase symmetry is just a part of the electromagnetic gauge symmetry, 


$$
\begin{aligned}
& \psi_{\alpha} \rightarrow e^{i f^{\alpha}(x)} \psi_{\alpha}, \\
& A_{\mu}^{\alpha} \rightarrow A_{\mu}^{\alpha}-\frac{1}{e} \partial_{\mu} f^{\alpha}(x), \\
& A_{z} \rightarrow A_{z}-\frac{1}{e d}\left(f^{1}-f^{2}\right),
\end{aligned}
$$

which can by no means be broken explicitly. The way to make the tunneling term gauge invariant is to introduce a path-dependent factor as in $\psi_{1}^{\dagger} \exp \left[\right.$ ie $\left.\int_{2}^{1} d \mathbf{x} \cdot \mathbf{A}\right] \psi_{2}$. We assume that the system is uniform in the perpendicular (z) direction between the two layers. Hence, we replace the integration and the derivative in the $z$ direction as follows:

$$
\int d z A_{z} \rightarrow d A_{z}, \quad \partial_{z} A_{\mu} \rightarrow \frac{1}{d} \Delta A_{\mu},
$$

where $d$ is the distance between the two layers. When the path is taken perpendicular to the layers, we obtain the tunneling interaction term as in (3.17), which is invariant under the phase transformation (3.11) together with the gauge transformation $A_{z} \rightarrow A_{z}-(1 / e d) 2 \Lambda$.

In the pseudospin language this is a kind of the local rotational symmetry around the $z$ axis in the pseudospin space. Although this local symmetry has never been addressed by other authors $[15,13]$, it plays important roles in various Josephson phenomena in the presence of the parallel magnetic field.

We now make a detailed analysis of the $\mathrm{QH}$ states as the condensed states of the bosonized electrons, temporarily switching off the voltage $\left(A_{0}^{\alpha}=0\right)$ and neglecting the tunneling interaction $(\lambda=0)$. The $\mathrm{QH}$ states satisfy the LLL condition,

$$
\left(D_{x}^{\alpha}-i D_{y}^{\alpha}\right) \psi_{\alpha}|f\rangle=0,
$$

or

$$
\left(D_{1}^{\alpha}-i D_{2}^{\alpha}\right)\left\langle\psi_{\alpha}\right\rangle=0,
$$

in the mean-field approximation. By solving the LLL condition, we find that there are many $\mathrm{QH}$ states with uniform densities $\rho_{\alpha}$ :

$$
\begin{aligned}
& \left\langle a_{k}\right\rangle=e A_{k}, \quad\left\langle\psi_{\alpha}\right\rangle=\sqrt{\rho_{\alpha}} e^{i \theta_{\alpha}}, \\
& \partial_{k} \theta+e \Delta A_{k}=0,
\end{aligned}
$$

where $\theta=\theta_{1}-\theta_{2}$ is the phase difference and $\Delta A_{k}=$ $A_{k}^{1}-A_{k}^{2}$ is the potential difference. We call these condensed states as QH states. Due to the constraint equation (3.14) these states are realized only at the magic filling factor

$$
\nu \equiv \frac{2 \pi \rho}{e B_{\perp}}=\frac{1}{m},
$$

with $\rho=\rho_{1}+\rho_{2}$. This equation fixes the total electron density in terms of the external magnetic field, but the density difference $\Delta \rho=\frac{1}{2}\left(\rho_{1}-\rho_{2}\right)$ is arbitrary. All these states are degenerate without the capacitance energy,

$$
E_{C}=\frac{e^{2}(\Delta N)^{2}}{2 C},
$$

which follows from the Hamiltonian (3.38) below, where $\Delta N=\int d^{2} x \Delta \rho$ and $C$ is the capacity of the double layers. By minimizing the capacitance energy, the ground state is uniquely determined to be

$$
\begin{aligned}
& \left\langle a_{k}\right\rangle=e A_{k}, \quad\left\langle\psi_{\alpha}\right\rangle=\sqrt{\rho_{0}} e^{i \theta_{\alpha}}, \\
& \partial_{k} \theta+e \Delta A_{k}=0,
\end{aligned}
$$

where $e \rho_{0}$ is the background positive charge density chosen identical on both of the two layers.

We may view the appearance of the Goldstone mode as follows. Since the capacity is proportional to the surface area $V$ of the layer, the capacitance energy (3.30) vanishes as $V \rightarrow \infty$ when $S_{z}$ is fixed. Hence, when we move a few electrons from one layer to the other, the resulting state is precisely degenerate with the ground state (3.31) in a system with $V \rightarrow \infty$, which guarantees the existence of the Goldstone mode. In our CS theory this degeneracy in the QH states has been proved [5] by using the Gaussian approximation around the solution (3.31) and by diagonalizing the Coulomb interaction (3.6) within the LLL states. Note that such a degeneracy never occurs in the state with $m \neq n$, where the constraint conditions (3.2) fix the electron numbers in each layers uniquely as $\rho_{1}=\rho_{2}=\rho_{0}$, and hence the transfer of electrons between the two layers inevitably create quasiparticles or quasiholes with their nontrivial Coulomb energies.

In the pseudospin language each $\mathrm{QH}$ state

$$
\left\langle\psi_{1}\right\rangle=\sqrt{\rho_{1}} e^{i \theta / 2}, \quad\left\langle\psi_{2}\right\rangle=\sqrt{\rho_{2}} e^{-i \theta / 2},
$$

corresponds to the pseudospin with its components $\left\langle S_{x}\right\rangle \propto \sin \theta,\left\langle S_{y}\right\rangle \propto \cos \theta$ and $\left\langle S_{z}\right\rangle \propto \Delta \rho$. Especially. after incorporating the capacitance energy, the ground state (3.31) corresponds to a ferromagnet with the direction of the pseudospin spontaneously chosen as

$$
\langle\theta\rangle=\theta_{0}, \quad\left\langle S_{z}\right\rangle=0,
$$

with $\theta_{0}$ a constant. Furthermore, the introduction of the tunneling interaction aligns the direction of the magnetization $\langle\mathbf{S}\rangle$ with the $y$ axis:

$$
\langle\theta\rangle=0, \quad\left\langle S_{z}\right\rangle=0 .
$$

Quantum mechanically the pseudospin rotates around this mean value with a nonzero frequency. This rotation is induced by the capacitance energy $\left[\propto(\Delta \rho)^{2}\right]$ and the tunneling energy $(\alpha \cos \theta)$ with the canonical conjugate variables $\theta$ and $\Delta \rho$; see later for more details.

In the rest of this paper we analyze the DLQH system with $m=n$ in the presence of the weak tunneling and capacitance effects. We also introduce the external parallel magnetic field $B_{k}^{\|}$and the external voltage $A_{0}^{\alpha}$ between the layers. By introducing these fields we can control the direction of the magnetization $\langle\mathbf{S}\rangle$ as well as the frequency of the rotational mode around it. 


\section{Low energy dynamics of $\theta$ and $\Delta N$}

Our main concern is the study of the low energy physics associated with the coherent tunneling between the two layers. This collective mode is described by the small fluctuation in the density $\Delta \rho \equiv \rho_{1}-\rho_{0}\left(=\rho_{0}-\rho_{2}\right)$ and its canonical conjugate phase difference $\theta \equiv \theta_{1}-\theta_{2}$. Let us parametrize

$$
\psi_{1}=\sqrt{\rho_{0}+\Delta \rho} e^{i \theta / 2}, \quad \psi_{2}=\sqrt{\rho_{0}-\Delta \rho} e^{-i \theta / 2},
$$

and treat the dynamical variables $\Delta \rho$ and $\theta$ in the mean field approximation. (Fluctuations of $\rho_{1}+\rho_{2}$ and its conjugate phase $\theta_{1}+\theta_{2}$ generate nontrivial Coulomb energy of pseudoparticles or pseudoholes; thus, it is irrelevant in our consideration of sufficiently low energy phenomena associated with the tunneling. The energy scale we are concerned about is much smaller than the average Coulomb energy between electrons.) The equations of motion of these variables govern the dynamics of the $\mathrm{QH}$ system under the influence of the parallel magnetic field $B_{k}^{\| l}$ and/or the voltage $\Delta A_{0}=A_{0}^{1}-A_{0}^{2}$.

In the pseudospin language, by the parametrization (3.35) the pseudospin components read

$$
\begin{aligned}
& S_{x}=-\int d^{2} x \sqrt{\rho_{0}^{2}-(\Delta \rho)^{2}} \sin \theta, \\
& S_{y}=\int d^{2} x \sqrt{\rho_{0}^{2}-(\Delta \rho)^{2}} \cos \theta \\
& S_{z}=\int d^{2} x \Delta \rho .
\end{aligned}
$$

This demonstrates that $\theta$ represents the direction of the pseudospin $\mathbf{S}$ projected onto the $x y$ pseudospace. We are interested in the dynamics of the ferromagnet state in which two components $S_{x}$ and $S_{y}$ are large $\left(\propto N_{0}\right)$ but $S_{z}$ is very small $(\propto \Delta N)$. The ferromagnet state is specified with the two variables $\theta$ and $\Delta \rho$.

In order to derive the equations of motion, first we examine the LLL constraint (3.27), which yields

$$
\partial_{k} \theta+e \Delta A_{k}=0,
$$

when the parametrization (3.35) is used and $\Delta \rho$ is assumed not to depend on the spatial coordinates. This equation implies that the spatial variation of the phase difference $\theta$ is controled by the parallel magnetic field $B_{k}$. (In the gauge $A_{z}=0$ it follows that $B_{k}=\varepsilon_{k j} \partial_{j} \theta / e d$.)

We next substitute the parametrization (3.35) into the Hamiltonian (3.17) and use the constraint equation (3.14). We derive the effective Hamiltonian describing the low energy phenomana as

$$
\mathcal{H}=-e \Delta A_{0} \Delta \rho-2 \lambda \rho_{0}\left(1-\frac{(\Delta \rho)^{2}}{2 \rho_{0}^{2}}\right) \cos \left(\theta+e d A_{z}\right),
$$

where the LLL constraint (3.37) is understood. In deriving (3.38) we have expanded the density as

$$
\sqrt{\rho_{1} \rho_{2}}=\sqrt{\left(\rho_{0}+\Delta \rho\right)\left(\rho_{0}-\Delta \rho\right)}=\rho_{0}\left(1-\frac{(\Delta \rho)^{2}}{2 \rho_{0}^{2}}\right)
$$

up to the order of $\left(\Delta \rho / \rho_{0}\right)^{2}$.

In order to incorporate the constraint (3.37) consistently, we consider the Lagrangian density by introducing a Lagrange multiplier $\zeta_{k}$ :

$$
\begin{aligned}
\mathcal{L}= & \Delta \rho\left(\partial_{t} \theta+e \Delta A_{0}\right)-\zeta_{k}\left(\partial_{k} \theta+e \Delta A_{k}\right) \\
& +2 \lambda \rho_{0}\left(1-\frac{(\Delta \rho)^{2}}{2 \rho_{0}^{2}}\right) \cos \left(\theta+e d A_{z}\right)
\end{aligned}
$$

where we have used the fact that $\Delta \rho$ and $\theta$ are the canonical conjugate each other. Now, the equations of motion are derived as stationary conditions of the Lagrangian:

$$
\begin{aligned}
& \partial_{t} \Delta \rho=\partial_{k} \zeta_{k}-2 \lambda \rho_{0} \sin \left(\theta+e d A_{z}\right) \\
& \partial_{t} \theta+e \Delta A_{0}=\frac{2 \lambda \Delta \rho}{\rho_{0}} \cos \left(\theta+e d A_{z}\right)
\end{aligned}
$$

together with constraint (3.37).

The behavior of the $\mathrm{QH}$ system is determined by the field equations (3.41), (3.42) and the LLL constraint (3.37). Let us study the physical meaning of these two equations and of the Lagrange multiplier $\zeta_{k}$. First, (3.42) describes that the time evolution of the phase difference $\theta$ is controled by the energy change (electrochemical potential difference) due to the movement of electrons from one layer to the other layer; the term $-e \Delta A_{0}$ is associated with the electric potential difference while the term proportional to $\lambda$ is associated with the tunneling energy. On the other hand, (3.41) represents the charge-current conservation. In order to see this, we consider the charge and currents implied by the Lagrangian (3.40):

$$
\begin{aligned}
& \Delta J_{0}=-\frac{\delta \mathcal{L}}{\delta \Delta A_{0}}=-e \Delta \rho, \\
& \Delta J_{k}=\frac{\delta \mathcal{L}}{\delta \Delta A_{k}}=-e \zeta_{k}, \\
& J_{z}=\frac{1}{d} \frac{\delta \mathcal{L}}{\delta A_{z}}=-2 e \lambda \rho_{0} \sin \left(\theta+e d A_{z}\right) .
\end{aligned}
$$

We now relate these to the charge and currents on each layer. Since the charge $J_{0}^{\alpha}$ on each layer is given by

$$
J_{0}^{1}=-J_{0}^{2}=-e \Delta \rho,
$$

it is clear that $\Delta J_{0}$ defines their difference:

$$
\Delta J_{0}=\frac{1}{2}\left(J_{0}^{1}-J_{0}^{2}\right) .
$$

Similarly, $\Delta J_{k}$ defines the difference of the currents $J_{k}^{\alpha}$ flowing on the two layers:

$$
\Delta J_{k}=\frac{1}{2}\left(J_{k}^{1}-J_{k}^{2}\right)
$$

We assume that there is no Hall current on the layers: 


$$
\sum_{\alpha} J_{k}^{\alpha}=0
$$

Then, we obtain

$$
J_{k}^{1}=-J_{k}^{2}=-e \zeta_{k} .
$$

Now, it is seen that the charge-current conservation on each layer,

$$
\partial_{t} J_{0}^{1}=\partial_{k} J_{k}^{1}-J_{z}, \quad \partial_{t} J_{0}^{2}=\partial_{k} J_{k}^{2}+J_{z},
$$

is reduced to the field equation (3.41) with use of (3.45), (3.46) and (3.50). This form of conservation law is understood by noting that the current flowing perpendicular to the layers does not exist outside the double layers.

From (3.50) the Lagrange multiplier $\zeta_{k}$ represents the current $J_{k}^{\alpha}$ flowing on the layers. As is well known, the LLL condition automatically leads to the divergentless currents, $\partial_{k} J_{k}^{\alpha}=0$, on each layers $\alpha=1,2$ : This means that $\partial_{k} \zeta_{k}=0$. However, in the presence of the parallel magnetic field we can have non-divergentless currents, just as we have the non-divergentless Hall current in the presence of an electric field. We expect that the field $\zeta_{k}$ is expressed in terms of the parallel magnetic field. This can be shown as follows.

We analyze the Maxwell equations relating the currents and the magnetic fields:

$$
-\varepsilon \partial_{t} E_{k}+\varepsilon_{k j}\left(\partial_{j} B_{z}-\partial_{z} B_{j}\right)=4 \pi J_{k},
$$

where the current $J_{k}$ exists only on the layers,

$$
J_{k}=J_{k}^{1} \delta\left(z-z_{1}\right)+J_{k}^{2} \delta\left(z-z_{2}\right),
$$

while $J_{z}$ is given by (3.45). Here, $z_{\alpha}$ are the $z$ coordinates of the layers, $z_{1}>z_{2}$.

We are concerned with the physical situation in which there are no parallel electric field $\left(E_{k}=0\right)$ : this corresponds to the condition (3.49) that there is no Hall current. We also assume that the perpendicular magnetic field is uniform in the $x y$ plane $\left(\partial_{k} B_{z}=0\right)$. Then, the Maxwell equation (3.52) is reduced to

$$
\partial_{z} B_{k}=4 \pi \varepsilon_{k \ell} J_{\ell}
$$

Integrating this equation with $J_{k}^{1}=-J_{k}^{2}=-e \zeta_{k}$, we obtain

$$
\begin{aligned}
B_{k}(x, t)= & -4 \pi e \varepsilon_{k \ell} \zeta_{\ell}(x, t)\left[\theta\left(z-z_{1}\right)-\theta\left(z-z_{2}\right)\right] \\
& +B_{k}^{\|}(x, t),
\end{aligned}
$$

where $\theta\left(z-z_{\alpha}\right)$ is a step function; $B_{k}^{\|}$is an "integration constant" representing the parallel magnetic field outside the both layers, that is, for $z>z_{1}$ or $z<z_{2}$. Hence, we identify $B_{k}^{\| l}$ as the external parallel magnetic field. The parallel magnetic field inside the layers $\left(z_{1}>z>z_{2}\right)$ is given by

$$
B_{k}(x, t)=4 \pi e \varepsilon_{k \ell} \zeta_{\ell}+B_{k}^{\prime \prime} .
$$

This equation is the desirable one which relates the current $\zeta_{\ell}$ with the parallel magnetic field $B_{k}$ between the layers. The external field $B_{k}^{\|}$induces the screening current $\zeta_{k}$ which reduces $B_{k}^{\|}$to $B_{k}$. It follows that without the parallel magnetic field the current $\zeta_{k}$ is divergentless $\left(\partial_{k} \zeta_{k}=0\right)$, as is expected.

In what follows, let us choose the gauge $A_{z}=0$, where we have

$$
B_{k}=-\varepsilon_{k \ell} \frac{\Delta A_{\ell}}{d} \quad E_{z}=-\frac{1}{d} \Delta A_{0} .
$$

These fields can be expressed in terms of the dynamical variables $\Delta \rho$ and $\theta$. Using the LLL constraint (3.37) we find

$$
B_{k}=-\varepsilon_{k \ell} \frac{\Delta A_{\ell} \cdot}{d}=\varepsilon_{k \ell} \frac{\partial_{\ell} \theta}{e d} .
$$

From this equation and (3.56) we obtain

$$
\zeta_{k}=\frac{1}{4 \pi e^{2} d} \partial_{k} \theta+\frac{\varepsilon_{k \ell}}{4 \pi e} B_{\ell}^{\|} .
$$

This is the equation determining the Lagrange multiplier field $\zeta_{k}$ in terms of the phase difference $\theta$ and the external parallel magnetic field $B_{k}^{\|}$. Physically, it represents a drift current due to the parallel magnetic field and does not depend on the electron mass $M$ as expected.

On the other hand, we have nonvanishing electric field $E_{z}$ between the two layers, which is controlled by the Maxwell equation,

$$
\varepsilon \partial_{z} E_{z}=-4 \pi J_{0}
$$

where

$$
J_{0}=J_{0}^{1} \delta\left(z-z_{1}\right)+J_{0}^{2} \delta\left(z-z_{2}\right),
$$

with (3.46). Integrating (3.60) we obtain an expected formula for the electric field generated by the charging $e \Delta \rho$ on the layers:

$$
E_{z}=\frac{4 \pi e \Delta \rho}{\varepsilon},
$$

or the electric potential difference:

$$
\Delta A_{0}=-\frac{4 \pi e d \Delta \rho}{\varepsilon} .
$$

Thus, the DL system has an electric capacity

$$
C=\frac{\varepsilon V}{4 \pi d},
$$

as it should be.

We now arrive at a closed set of equations governing the dynamics of the density difference $\Delta \rho$ and its conjugate phase difference $\theta$. Substituting (3.59) and (3.63) into our basic equation (3.41) and (3.42), we obtain 


$$
\begin{aligned}
& \partial_{t} \Delta \rho=\frac{1}{4 \pi e^{2} d}\left(\partial_{x}^{2} \theta+\partial_{y}^{2} \theta\right)-2 \lambda \rho_{0} \sin \theta \\
& \partial_{t} \theta=\frac{4 \pi e^{2} d \Delta \rho}{\varepsilon}+\frac{2 \lambda \Delta \rho}{\rho_{0}} \cos \theta
\end{aligned}
$$

The phase $\theta$ and the density $\Delta \rho$ are determined by solving these equations with an appropriate boundary condition.

We note that the Maxwell equations are used only to determine the Lagrange multiplier field $\zeta_{k}$ and the electric potential $\Delta A_{0}$. When there is no parallel magnetic field, we have $\zeta_{k}=0$ and hence the set of equations (3.65) and (3.66) contain no more information than the set of our basic equations (3.41) and (3.42), provided that the expression (3.63) for the potential $\Delta A_{0}$ is taken for granted. The Maxwell equations play an important role only when the parallel magnetic field exists.

In the pseudospin formalism the effective Hamiltonian (3.38) reads

$$
H=\int d^{2} x \mathcal{H}=-e \Delta A_{0} S_{z}-h S_{y},
$$

where we have chosen the gauge $A_{z}=0$. When the parallel magnetic field is neglected, it is easy to derive the equations of motion. Using the commutation relation, $\left[S_{i}, S_{j}\right]=i \varepsilon_{i j k} S_{k}$, we may evaluate the Heisenberg equations of motion, $\partial_{t} S_{j}=i\left[H, S_{j}\right]$, as

$$
\begin{aligned}
& \partial_{t} S_{z}=h S_{x}, \\
& \partial_{t} S_{x}=e \Delta A_{0}^{\text {ext }} S_{y}-\frac{e^{2}}{C}\left(S_{y} S_{z}+S_{z} S_{y}\right)-h S_{z}, \\
& \partial_{t} S_{y}=-e \Delta A_{0}^{\text {ext }} S_{x}+\frac{e^{2}}{C}\left(S_{x} S_{z}+S_{z} S_{x}\right),
\end{aligned}
$$

with $\Delta A_{0} \equiv \Delta A_{0}^{\text {ext }}-(e / C) S_{z}$. These equations are identical to our basic equations (3.41) and (3.42) with $\partial_{k} \zeta_{k}=0$, when we approximate the expressions (3.36a) $\sim(3.36 \mathrm{c})$ of the pseudospin $\mathbf{S}$ by expanding the square root up to the order of $\left(\Delta \rho / \rho_{0}\right)^{2}$. It is obvious from these equations that, when the external voltage $\Delta A_{0}^{\text {ext }}$ $\left(=V_{\text {ext }}\right)$ is applied, the pseudospin $\mathbf{S}$ rotates around the $z$ axis with its frequency $e V_{\text {ext }}$, provided that the charging $\Delta N\left(=S_{z}\right)$ vanishes by attaching external leads to each layers but with $\partial_{t} S_{z} \neq 0$ by feeding the current externally. As we discuss in the succeeding section, this is the Josephson effect with DC voltage feed.

\section{JOSEPHSON PHENOMENA}

Using the formulas we have presented in the previous section we proceed to discuss various Josephson phenomena in DLQH system. The basic origin of the Josephson phenomena is the existence of many condensed QH states (3.28) at the filling factor $\nu=1 / m$. They are coherent states of electrons, having various density difference $\Delta \rho=\frac{1}{2}\left(\rho_{1}-\rho_{2}\right)$ with $\rho_{1}+\rho_{2}$ fixed. They are degenerate if the tunneling and the capacitance energies are neglected. In actual system without external leads there is no degeneracy in these $\mathrm{QH}$ states due to the charging $(\Delta \rho \neq 0)$ effect. If the external leads are attached to the system, tunneled electrons flow out of the system without charging $(\Delta \rho=0)$, which leads to the degeneracy of the states. The key point of the Josephson phenomena is that electrons can make a coherent tunneling between the layers, connecting these QH states, whether the degeneracy exists or not.

\section{A. Josephson effect}

We analyze the Josephson effect by attaching external leads to the system. However, let us first argue how the present system is similar to or different from the well known superconductor Josephson junction [12] in the case without external leads. When the capacitance and tunneling energies are neglected, the QH states $\Psi\left(N_{0}, N_{0}\right)$ and $\Psi\left(N_{0}+\Delta N, N_{0}-\Delta N\right)$ are degenerate each other. The degeneracy is resolved by the capacitance and tunneling energies. The energy of the system is given by the Hamiltonian (3.38), or

$$
E=E_{C}+E_{T}+E_{\Delta}
$$

with

$$
\begin{aligned}
& E_{C}=\frac{(e \Delta \rho V)^{2}}{2 C}, \\
& E_{T}=-2 \lambda \rho_{0} V \cos \theta, \\
& E_{\Delta}=\frac{\lambda(\Delta \rho)^{2}}{\rho_{0}} V \cos \theta,
\end{aligned}
$$

where $C$ is the electric capacity (3.64). It is a special feature that the tunneling interaction in (3.17) produces two terms $E_{T}$ and $E_{\Delta}$.

Let us tempolarily neglect the term $E_{\Delta}$. Then, the QH system is essentially the same as the superconductor Josephson junction, where the system is described with the capacitance and the tunneling energies, $E_{C}$ and $E_{T}$. Also in the present case the capacitance energy $E_{C}$ is much smaller than the tunneling energy $E_{T}$ for typical values of $\lambda, \rho_{0}$ and $C$, as in the superconductor case. Hence, the degeneracy is resolved mainly by the tunneling energy $E_{T}$, and the states $\Psi\left(N_{1}, N_{2}\right)$ are mixed among themselves:

$$
\Psi(\theta)=\sum_{\Delta N} e^{i \theta \Delta N} \Psi\left(N_{0}+\Delta N, N_{0}-\Delta N\right),
$$

where $\theta$ is the phase difference in (3.35). Without the capacitance energy, the eigen states would be given by the states (4.5) and specified by $\theta$ with the continuous energy spectrum (4.3) with no gap. Actually, the capacitance energy $E_{C}$ exists. Since $E_{C}$ depends on $\Delta \rho$, which is the canonical conjugate to $\theta$, the states are rearranged so that the energy eigen states have discrete energy spectra; namely, the system acquires a gap, 


$$
\omega_{J} \equiv \sqrt{\frac{8 \pi e^{2} d \rho_{0} \lambda}{\varepsilon}},
$$

as we see in (4.37) below. These are the well known features in superconductor Josephson junction [12].

In the pseudospin language, when the capacitance energy is neglected the states $\Psi\left(N_{0}+\Delta N, N_{0}-\Delta N\right)$ are degenerate with respect to the pseudospin component $S_{z}=\Delta N$. The new state $\Psi(\theta)$ is constructed with a superposition of the eigen states of $S_{z}$ as in (4.5), and it represents the state which has a magnetization with the angle $\theta$. Now, the tunneling effect is equivalent to applying the pseudo-magnetic field in the $y$ direction, which aligns the pseudospin direction with the $y$ axis. This is the ground state of the ferromagnet described by $\Psi(\theta=0)$ with the minimum energy $E_{T}(\theta=0)$. The inclusion of the capacitance energy $E_{C}$ makes the pseudospin rotate around the $y$ axis with frequency (4.6).

On the other hand, when we attach external leads, the system turns out to have a continuous spectrum; namely, oscillations with any low frequency are allowed. This is because there is no charging $(\Delta \rho=0)$ in the presence of external leads, and hence the capacitance term $E_{C}$ is ineffective.

We now examine the effect due to the term $E_{\Delta}$, which may be interpreted as a chemical potential difference induced by the charging $\Delta \rho$. When no external leads are attached to the system, this term opens a new tunneling gap $\Delta_{\mathrm{SAS}}(=2 \lambda)$ which is absent in superconductor Josephson junction. (We analyze this gap in detail in the succeeding subsection.) Due to this term there is an incorrect argument [13] with respect to the Josephson effect. The argument reads as follows [13]: Even if external leads are attached and $E_{C}$ is ineffective, the term $E_{\Delta}$ gives rise to a tunneling gap $\left(\Delta_{\mathrm{SAS}}\right)$ of the system, and does not allow Josephson behaviors, for instance, by inhibiting the low frequency oscillation in the Josephson current $(\partial \Delta \rho / \partial t)$ in the DC-voltage Josephson circuit. Obviously, this argument is incorrect. It is clear that the term $E_{\Delta}$ becomes also ineffective when there is no charging $(\Delta \rho=0)$ because $E_{\Delta}$ vanishes just as $E_{C}$ does. The role of the term $E_{\Delta}$ is essentially the same as that of $E_{C}$ in the Josephson effect. Furthermore, in actual systems we can check that $E_{\Delta}$ is numerically about ten times smaller than $E_{C}$. Therefore, the term $E_{\Delta}$ is harmless to the QH-state Josephson effect just as the capacitance term $E_{C}$ is harmless.

The origin of the confusion would probably be that the energy terms $E_{C}, E_{T}$ and $E_{\Delta}$ are not identified separately in their approach [13] using fermion language or equivalent pseudospin language, and thus one cannot make a clear comparison with the standard formulas in superconductor Josephson junction. On the contrary, our formulation based on the CS gauge theory produces various formulas quite similar to the standard ones, and consequently the roles of each terms describing the low energy behavior of the QH states become transparent.
Indeed, we can examine the problem by using the field equations. The basic equations are given by (3.41) and (3.42), or

$$
\begin{aligned}
& \partial_{t} \Delta \rho=-2 \lambda \rho_{0} \sin \theta, \\
& \partial_{t} \theta=-e \Delta A_{0}+\frac{8 \lambda \Delta \rho}{\rho_{0}} \cos \theta,
\end{aligned}
$$

where we have taken the gauge $A_{z}=0$ and assumed no parallel magnetic field to exist $\left(\zeta_{k}=0\right)$. When external leads are attached to the system, there exists no charging $(\Delta \rho=0)$. Hence, the electric potential difference $\Delta A_{0}$ is simply given by the external voltage, and the chemical potential term $E_{\Delta}$ does not contribute in (4.8):

$$
\partial_{t} \theta=-e \Delta A_{0}=e V_{\mathrm{ext}} .
$$

By way of external leads, electrons flow into the system and flow out of the system $\left(\partial_{t} \Delta \rho \neq 0\right)$, with the tunneling current being given by (3.44) or

$$
J_{z}=e \partial_{t} \Delta \rho=-2 e \lambda \rho_{0} \sin \theta .
$$

In this way our equations are just the famous ones describing the Josephson behavior in superconductor. We conclude that the Josephson current (4.10) flows between the two layers with the phase $\theta$ being controlled by (4.9).

In particular, in a $\mathrm{QH}$ system with DC-voltage feed, we obtain that

$$
\theta=e V_{\mathrm{ext}} t
$$

Because electrons tunnel in the presence of voltage $\left(V_{\text {ext }} \neq 0\right)$, they acquire energy, which is emitted as electromagnetic radiation. The frequency is $e V_{\text {ext }}$, which is one half of that in superconductor Josephson junction. This shows that the condensate in the QH state is the unpaired electron and not the Cooper pair.

On the other hand, in a $\mathrm{QH}$ system with $\mathrm{DC}$-current feed $\left(J_{z}=\right.$ constant $\left.<2 e \lambda \rho_{0}\right)$, the phase difference is fixed to be a constant $\left(\theta=\theta_{0}\right)$ by (4.10). Then, from (4.9) it follows that $V_{\mathrm{ext}}=0$, as in the superconductor case. Therefore, the tunneling current is a superconducting current.

We next consider a $\mathrm{QH}$ system with DC-voltage feed in a strong external parallel magnetic field $B_{\|}$, which we take in the $y$ direction. When $B_{\|}$is too strong, it penetrates into the junction freely. This is the case

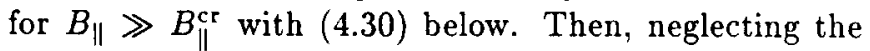
screening effect $\left(\zeta_{k}=0\right.$ or $\left.B_{y}=B_{\|}\right)$, we may solve (4.9) and (3.58) as

$$
\theta=e V_{\text {ext }} t-x e d B_{\|} .
$$

The Josephson current oscillates in space and time:

$$
J_{z}=-2 e \lambda \rho_{0} \sin \left(e V_{\mathrm{ext}} t-x e d B_{\|}\right) .
$$

We may also consider a $\mathrm{QH}$ system with $\mathrm{DC}$-current feed in a strong external parallel magnetic field $B_{\|}$. In this 
case we may solve (4.9) with $V_{\text {ext }}=0$ and (3.58) by neglecting the screening effect:

$$
\theta=\theta_{0}-x e d B_{\|}
$$

and hence

$$
J_{z}=-2 e \lambda \rho_{0} \sin \left(\theta_{0}-x e d B_{\|}\right) .
$$

Evaluating the total Josephson current we obtain the Fraunhofer pattern:

$$
J_{\text {total }}=\ell \int d x J_{z}(x)=-2 e \lambda \rho_{0} \ell^{2} \sin \delta_{0} \frac{\sin \left(\pi \Phi / \Phi_{0}\right)}{\pi \Phi / \Phi_{0}},
$$

where $\ell$ is the size of the junction: $\Phi=B_{\|} d \ell$ is the total parallel magnetic flux penetrated in the junction and $\Phi_{0}=2 \pi / e$ is the unit of tlux.

In the pseudospin language we may understand how the term $E_{\Delta}$ arizes in the tunneling interaction $H_{T}=$ $-h S_{y}$ in the following way. For a sufficiently small value of $S_{z}(\propto \Delta \rho)$ we may expand $S_{y}$ in $(3.36 \mathrm{~b})$ as

$$
S_{y}=S_{y}^{0}-\frac{S_{z}^{2}}{2 N_{0}^{2}} S_{y}^{0}
$$

in terms of the pseudospin $\mathbf{S}^{0}=\left(S_{x}^{0}, S_{y}^{0}\right)$ of the XY model, where

$$
S_{x}^{0}=N_{0} \sin \theta, \quad S_{y}^{0}=N_{0} \cos \theta .
$$

The last term of (4.17) yields $E_{\Delta}$. This pseudospin $\mathbf{S}^{0}$ is subject to the constraint:

$$
\left(S_{x}^{0}\right)^{2}+\left(S_{y}^{0}\right)^{2}=N_{0}^{2}
$$

Now, the Josephson equations (4.9) and (4.10) are rewritten as

$$
\partial_{t} S_{z}=h S_{x}^{0}, \quad \partial_{t} S_{x}^{0}=e V_{\mathrm{ext}} S_{y}^{0} \quad \partial_{t} S_{y}^{0}=-e V_{\mathrm{ext}} S_{x}^{0}
$$

For a constant nonvanishing $V_{\text {ext }}$ the pseudospin rotates around the $z$ axis with its frequency $e V_{\text {ext }}$. This is the DC-voltage Josephson effect. Such a rotation is possible because the external leads suppress the charging $\left(S_{z}=0\right)$ but keeps $\partial_{t} S_{z}$ nonvanishing. On the other hand, the DC- current Josephson effect may be understood as follows. The pseudospin component $S_{x}$ can be controled by feeding a DC current across the layers. The direction of the pseudospin is fixed globally, $\langle\theta\rangle=\theta_{0}$, by fixing $S_{x}$.

However, the pseudospin behaves differently in the presence of a strong external parallel magnetic field. The strong external parallel magnetic field modulates the direction of the magnetization (S) locally as (4.12) in the DC-voltage circuit or (4.14) in the DC-current circuit. Then, the pseudospin component $S_{x}$ is given by (4.13) or (4.15) apart form a constant factor, which is the tunneling current.
In order to realize the Josephson effect experimentally, it is necessary to check that no charging $(\Delta \rho=0)$ occurs in the system. There might exist subtle problems in making a QH-state device with external leads because the layers themselves are not superconductor. To check the existence of the coherent tunneling current between the layers, it may be easier to detect its characteristic oscillation (plasmon) mode by using an isolated system, which we discuss later.

\section{B. Meissner effect}

As we have just studied, the external parallel magnetic field affects the tunneling current (or the pseudospin direction). However, when it is very weak, the parallel magnetic field can be screened by the superconducting current. This is the Meissner effect. We study this effect before discussing the oscillation mode of the tunneling current (or the rotational mode of the pseudospin).

The relevant equation for discussing the effect is given by (3.41) with (3.59), or the sine-Gordon equation:

$$
\partial_{x}^{2} \theta+\partial_{y}^{2} \theta-8 \pi e^{2} d \rho_{0} \lambda \sin \theta=0
$$

where we have set $\partial_{t} \Delta \rho=0$ because the Meissner effect is a static phenomena. In the static phenomena there is actually no charging $(\Delta \rho=0)$, since otherwise the induced voltage (3.62) makes $\theta$ evolve in time according to the basic formula (3.42). Now, (4.21) may be derived from the following Hamiltonian:

$$
H_{\text {static }}=\int d^{2} x\left(\frac{1}{8 \pi e^{2} d}\left[\left(\partial_{x} \theta\right)^{2}+\left(\partial_{y} \theta\right)^{2}\right]-2 \lambda \rho_{0} \cos \theta\right)
$$

which is equivalent to

$$
H_{\text {static }}=\int d^{2} x\left(\frac{d}{8 \pi}\left(B_{x}^{2}+B_{y}^{2}\right)-2 \lambda \rho_{0} \cos \theta\right),
$$

because of the formula (3.58) relating the parallel magnetic field $B_{k}$ to the phase field $\theta$. This static Hamiltonian consists of our original Hamiltonian (3.38) with $\Delta \rho=0$ and the energy of the parallel magnetic field.

We proceed to analyze the Meissner effect by applying the external magnetic field $B_{\|}$in the $y$ direction. Recall that the parallel magnetic field $B_{y}$ inside the junction is given by (3.56) and (3.58), or

$$
B_{y}=-\frac{1}{e d} \partial_{x} \theta=-4 \pi e \zeta_{x}+B_{\|} .
$$

It is to be observed that $B_{y}$ is solely determined by the phase field $\theta$ and that $\theta$ is subject to the static sine-Gordon equation (4.21). The ground- state solution $(\theta=0)$ of the Hamiltonian (4.22) corresponds to the completely screened solution. It is also observed that the screening of the parallel magnetic field $B_{\|}$is realized 
by the currents $J_{x}^{\alpha}= \pm e \zeta_{x}$ flowing on the layers. The physical picture reads as follows. Electrons first tunnel from one layer to the other, flow in the $x$ direction, tunnel back to the initial layer, and flow in the opposite $x$ direction. This circular motion of the drift current induced by the external parallel magnetic field $B_{\|}$screens the field $B_{\|}$itself. It is a kind of Landau diamagnetism.

In solving the static equation (4.21) we need to impose an appropriate boundary condition. The boundary condition is that the current $J_{x}^{\alpha}= \pm e \zeta_{x}$ should vanish at the edge of the layers, or

$$
B_{y}=-\frac{1}{e d} \partial_{x} \theta=B_{\|},
$$

at $x=0$ : We have taken the $x$ coordinate of the edge to be $x=0$. For a sufficiently small parallel magnetic, we can linearize the sine-Gordon equation (4.21) to obtain the London equation:

$$
\partial_{x}^{2} \theta=8 \pi e^{2} d \rho_{0} \lambda \theta
$$

The solution is given by

$$
\theta(x)=e d \ell_{V} B_{\|} e^{-x / \ell_{V}},
$$

or

$$
B_{y}(x)=B_{\|} e^{-x / \ell_{V}},
$$

where $\ell_{V}$ is the penetration depth,

$$
\ell_{V}=\frac{1}{\sqrt{8 \pi \lambda e^{2} d \rho_{0}}} .
$$

Obviously, the external field $B_{\|}$is screened completely except for the edge of the junction, where it penetrates within the penetration depth $\ell_{V}$. It should be mentioned that the completely screening solution exists only for sufficiently weak external field $B_{\|}$. We may estimate the critical field as

$$
B_{\|}^{\mathrm{cr}}=\frac{\pi}{e d \ell_{V}} .
$$

In actual samples we have $\ell_{V} \approx 0.03 \mathrm{~mm}$ and $B_{\|}^{\text {cr }} \approx 100$ Gauss.

When the parallel magnetic field increases, it penetrates into the junction gradually as sine-Gordon magnetic vortices, which are solutions of the sine-Gordon equation (4.21). It is notable that the magnetic flux $\Phi$ of the sine-Gordon vortex is quantized with the unit of

$$
\Phi_{0}=d \int d x B_{y}=\frac{1}{e} \int d x \partial_{x} \theta=\frac{2 \pi}{e},
$$

as reflects the condensation of unpaired electrons. Finally, when the magnetic field becomes sufficiently large $\left(B_{\|} \gg B_{\|}^{\text {cr }}\right)$, it penetrates into the junction freely. All these features are quite the same as in superconductor Josephson junction.
In the pseudospin language our result reads as follows. The parallel magnetic field $B_{\|}$acts on the pseudospin to change its axis at each point on the layer. However, when $B_{\|}$is sufficiently weak, its effect is cancelled by the screening current. Consequently, the ground state is still the ferromagnet with the axis of the magnetization parallel to the $y$ axis,

$$
\langle\theta\rangle=0,
$$

except for the vicinity of the edge of the layer, where the parallel magnetic field penetrates into the junction and modulates the axis by the angle (4.27). This is the Meissner effect. The Meissner effect is effective only for $B_{\|}<100$ Gauss.

\section{Anderson plasmon}

Let us analyze the low-lying collective excitations of the bosonized electron condensation (3.35). The excitations are oscillation modes of the charging $\Delta \rho$ and its conjugate phase difference $\theta$, which are induced by the coherent tunneling of electrons. Obviously, the charging $e \Delta \rho$ induces the electric field, which then acts to diminish the charging itself. Thus, the excitations look like plasma oscillations in electron gas. We call such oscillation modes as Anderson plasmons in analogy to the superconductor case. These oscillation modes are obtained by solving (3.65) and (3.66), or

$$
\begin{aligned}
\partial_{t} \Delta \rho & =\frac{1}{4 \pi e^{2} d}\left(\partial_{x}^{2} \theta+\partial_{y}^{2} \theta\right)-\Delta_{\mathrm{SAS}} \rho_{0} \sin \theta \\
\partial_{t} \theta & =\frac{4 \pi e^{2} d}{\varepsilon} \Delta \rho+\frac{\Delta_{\mathrm{SAS}}}{\rho_{0}} \Delta \rho \cos \theta .
\end{aligned}
$$

where $\Delta_{\mathrm{SAS}}$ is the tunneling gap given by $\Delta_{\mathrm{SAS}}=2 \lambda$.

For small fluctuations $\theta,(4.33)$ and (4.34) yield

$$
\partial_{t}^{2} \theta-\frac{1}{\varepsilon} \frac{\omega_{P}^{2}}{\omega_{J}^{2}}\left(\partial_{x}^{2} \theta+\partial_{y}^{2} \theta\right)+\omega_{P}^{2} \theta=0,
$$

where

$$
\omega_{P}=\sqrt{\omega_{J}^{2}+\Delta_{\mathrm{SAS}}^{2}},
$$

and

$$
\omega_{J}=\sqrt{\frac{4 \pi e^{2} d \rho_{0} \Delta_{\mathrm{SAS}}}{\varepsilon}} .
$$

The dispersion relation is given by

$$
\omega(k)^{2}=\frac{1}{\varepsilon} \frac{\omega_{P}^{2}}{\omega_{J}^{2}} k^{2}+\omega_{P}^{2} .
$$

Here, $\omega_{P}$ is the plasmon frequency. Note that in the case of a superconductor the plasmon frequency is simply given by $\omega_{J}$, but now it also receives a contribution from 
the tunneling gap $\Delta_{\text {SAS. }}$. This contribution originates in the energy term $E_{\Delta}$ in (4.4).

In the pseudospin language $\omega_{P}$ may be understood as the frequency with which the pseudospin rotates around the $y$ axis. As we have already noticed, the tunneling interaction is interpreted as the interaction with the external pseudo-magnetic field aligning the direction of the pseudospin with the $y$ axis. The capacitance and tunneling terms rotate the pseudospin around the $y$ axis with frequency $\omega_{P}$. It is an interesting question how the rotational mode of the pseudospin is affected by the strong external parallel magnetic field which modulates the direction of the magnetization. The corresponding problem is easily analyzed in our CS formalism.

Let us analyze the system where the parallel magnetic field is applied with its strength much larger than $B_{\|}^{\mathrm{cr}}$. Then, the screening effect can be neglected and we may set $\theta=\theta_{0}-x e d B_{\|}$as in (4.14). Here, we have taken the parallel magnetic field in the $y$ direction. Let us combine (4.33) and (4.34) to derive that

$$
\begin{aligned}
\varepsilon \partial_{t}^{2} \theta-\partial_{x}^{2} \theta-\partial_{y}^{2} \theta & +4 \pi e^{2} d \rho_{0} \Delta_{\mathrm{SAS}} \sin \theta \\
& =\frac{\varepsilon \Delta_{\mathrm{SAS}}}{\rho_{0}} \partial_{t}(\Delta \rho \cos \theta)
\end{aligned}
$$

We now consider a small uniform fluctuation $\chi$ by setting

$$
\theta=\theta_{0}-x e d B_{\|}+\chi
$$

with a constant $\theta_{0}$. We substitute this into (4.39). In so doing we use the Maxwell equation of the first kind, $\partial_{t} B_{y}=\partial_{x} E_{z}$. Substituting (3.58) and (3.62), or $B_{y}=$ $-\partial_{x} \theta / e d$ and $E_{z}=-\Delta A_{0} / d$, into this equation we find that $\partial_{x}\left(\partial_{t} \theta+e \Delta A_{0}\right)=0$. Then, (3.42) yields

$$
\partial_{x}(\Delta \rho \cos \theta)=0 \text {. }
$$

Because $\Delta p \cos \theta$ is independent of $x$, we may rewrite

$$
\partial_{t}(\Delta \rho \cos \theta)=\partial_{t}(\Delta \rho \cos \chi) \approx-\Delta_{\mathrm{SAS}} \rho_{0} \chi,
$$

for a sufficient small uniform fluctuation $\chi$, where use was made of (4.33). Therefore, we obtain

$$
\partial_{t}^{2} \chi+\Delta_{\text {SAS }}^{2} \chi+\omega_{J}^{2} \sin \left(\theta_{0}-x e d B_{\|}+\chi\right)=0 .
$$

Except for the term $\Delta_{\text {SAS }}^{2} \chi$ this is again a well known equation in superconductor Josephson junction with the magnetic field [12]. For the consistency of the assumption that $\chi$ is a uniform field, we take the average of this equation over the size of the junction [22], and obtain

$$
\partial_{t}^{2} x+\omega_{P}^{2} x=0
$$

where the plasmon frequency is given by

$$
\begin{aligned}
\omega_{P}(\Theta) & =\sqrt{\omega_{J}^{2}\left|\frac{\sin \left(\pi \Phi / \Phi_{0}\right)}{\pi \Phi / \Phi_{0}}\right|+\Delta_{\mathrm{SAS}}^{2}} \\
& =\sqrt{\omega_{J}^{2}\left|\frac{\sin \left(2 \pi d \ell \rho_{0} \tan \Theta\right)}{2 \pi d \ell \rho_{0} \tan \Theta}\right|+\Delta_{\mathrm{SAS}}^{2}},
\end{aligned}
$$

with $\Theta$ the tilt angle $\left(\tan \Theta=B_{\|} / B_{\perp}\right)$.

It is interesting to remark that the averaged Josephson current $\left(\bar{J}_{z}\right)$ is suppressed by the penetrated magnetic flux $\left(\sim \ell d B_{\|}\right)$and given by

$$
\bar{J}_{z} \sim \frac{1}{\ell} \int_{0}^{\ell} d x \sin \left(\theta_{0}-x e d B_{\|}\right) \propto \frac{1}{\ell d B_{\|}} .
$$

Because of this suppression of the tunneling current the plasmon frequency $\omega_{P}$ is reduced from $\omega_{P}=$ $\sqrt{\omega_{J}^{2}+\Delta_{\text {SAS }}^{2}}$ to the value as given by (4.45). In particular, when the total penetrated flux is just one unit of flux $\left(\Phi=\Phi_{0}=2 \pi / e\right)$, the plasmon frequency takes the minimum value $\left(\omega_{P}=\Delta_{\mathrm{SAS}}\right)$.

In the pseudospin language it turns out that the strong external parallel magnetic field rotates the direction of the magnetization locally around the pseudospin $z$ axis by the angle

$$
\langle\theta\rangle=\theta_{0}-x e d B_{\|},
$$

with $\theta_{0}$ a constant. This rotation effectively reduces the frequency of the pseudospin oscillation around its mean value.

\section{EXPERIMENTAL OBSERVATION OF ANDERSON PLASMONS}

It seems that the experimental indication of Josephson phenomena is most easily achieved by observing Anderson plasmons. In this section we point out that such plasmon excitations may have already been observed indirectly in a recent experiment [16]. We also propose some direct ways of observing them by microwave techniques.

Before discussing these matters, we wish to describe a physical picture of the realistic QH-state Josephson junction. We remember that, in a superconductor, because of the superfluid mode the coherence of the phase is maintained all over the sample. We may interpret this fact in the following way. Suppose that the superconductor is decomposed into many tiny domains and that each domains have different coherent phases. In such a situation the Josephson current flows inmediately between the adjacent domains and align their phases, enforcing one coherent phase all over the sample. On the contrary, in the QH state such a superconducting mode is absent on the layer. Thus, once many tiny domains are made, there is no reason that one coherent phase is introduced all over the sample. Now, in DL systems the interlayer tunneling acts to prevent the phase coherence of the condensate on the layer, and will break the $\mathrm{QH}$ system into tiny domains. In each domain the Josephson current flows between the layers, which enforces the phase coherence across the layers only within the domain. Namely, our physical assumption is that the QH state is made of an ensemble of small Josephson junctions with 
various sizes $\ell$. We call this size $\ell$ the "phase-coherence" length. Then, there exists the maximum size $\ell_{M}$ of the domain in each sample. In this section we consider the QH state only at $\nu=1$, i.e., $e B=2 \pi \rho=4 \pi \rho_{0}$.

We make a comment on the terminology. Do not confuse "phase-coherence length" with "coherent length" in superconductor. The latter means the length scale of the single Cooper pair, while the former means the length scale in which the phase of Cooper pairs (or bosonized electrons in our case) are aligned: the phase-coherent length of superconductor is infinite.

\section{A. Activation energy anomaly}

A recent experiment on a DLQH system [16] has revealed an anomalous behavior in the activation energy versus the parallel magnetic field in the $\mathrm{QH}$ states where the Josephson effect had been predicted. We have reproduced typical experimental data [16] in Fig.1, where the horizontal axis is the tilt angle $\tan \Theta=B_{\|} / B_{\perp}$. The main features of the anomaly are that in the vicinity of $\Theta=0$ it decreases rapidly as $\Theta$ increases: Apparently there exist a critical tilt angle $\Theta_{c}$, beyond which it becomes almost constant.

Recall that naively a smooth decrease of the activation energy is expected in DLQH states if it is given by the single-particle (SP) gap energy. As we have remarked before, when the energy gap between the SP symmetric and antisymmetric states becomes large, the $\mathrm{QH}$ state with $\nu=1$ is formed on the SP symmetric state. The activation energy is then given by this gap energy, which is shown to decrease smoothly as the parallel magnetic field increases. It behaves typically as

$$
E_{\mathrm{SAS}}(\Theta) \propto \exp \left[-\frac{1}{4}\left(\frac{d}{\ell_{B}}\right)^{2} \tan ^{2} \Theta\right]
$$

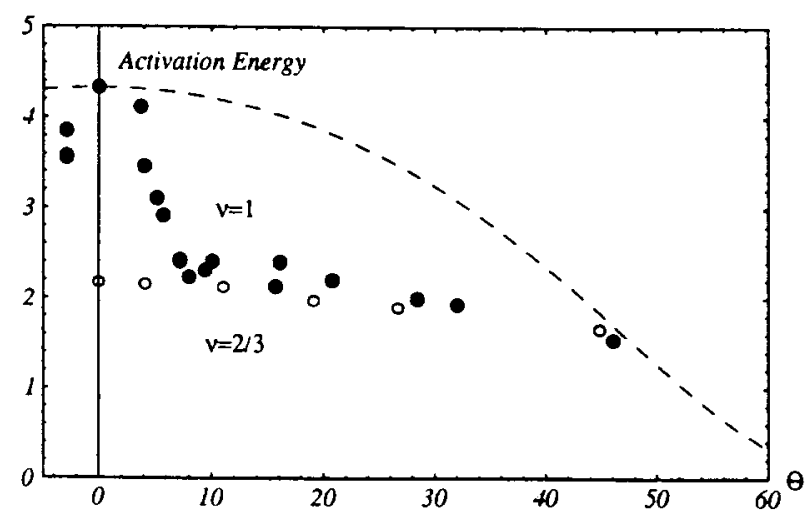

Fig.1: The measured activation energy as a function of the tilt angle. The solid circles are the $\mathrm{QH}$ state at $\nu=1$, while the open circles for the QH state at $\nu=2 / 3$. The dashed curve represents the activation energy implied by the SP gap formula with arbitrary normalization at $\Theta=0$.
We have plotted this curve also in Fig.1. (The normalization of $E_{\mathrm{SAS}}(\theta)$ at $\theta=0$ has been chosen arbitrarily to fit the data.) It is clear that the observed activation energy decreases much more rapidly than that expected by the SP gap formula (5.1).

This anomaly has so far been observed only in samples with $\nu=1$. For instance, it has not been observed in other DLQH states with $\nu=\frac{1}{2}, \frac{2}{3}$ etc., where the activation energy is almost independent of $\Theta$, which is also seen in Fig.1. Thus, it is. natural to associate it with the Josephson tunneling we have predicted. To be more specific we wish to interpret this anomaly in terms of Anderson plasmons. The plasmons are neutral, but they are oscillation modes of the charging of the electrons, $\Delta \rho=\Delta \rho_{0} \sin \left(\omega_{P} t\right)$, and of the electric field by way of (3.62) or

$$
E_{z}=\frac{4 \pi e \Delta \rho_{0} \sin \left(\omega_{P} t\right)}{\varepsilon}
$$

with $\omega_{P}$ the plasmon frequency (4.36). Therefore, plasmon excitations affect transport phenomena. In particular, the activation energy determined by measuring the electric resistivity $\rho_{x x}$ should detect their excitations.

In our physical picture, an actual sample is made of an ensemble of small Josephson junctions, in each of which Anderson plasmons with characteristic frequency may be excited. In general there are various junctions with different sizes $\ell$, in which the plasmon energy is given by $(4.45)$ or

$$
E(\Theta, \ell)=\sqrt{\omega_{J}^{2}\left|\frac{\sin \left(2 \pi d \ell \rho_{0} \tan \Theta\right)}{2 \pi d \ell \rho_{0} \tan \Theta}\right|+\Delta_{\mathrm{SAS}}^{2}}
$$

where $\Theta$ is the tilt angle, $\tan \Theta=B_{\|} / B_{\perp}$. The plasmon

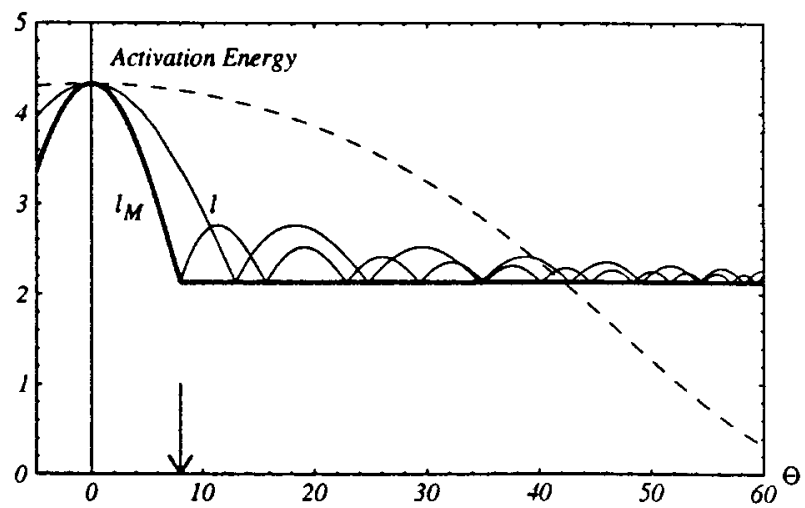

Fig.2: Two thin curves represent the plasmon gap formula (5.3) with two different size $\ell(=1700 \AA)$ and $\ell_{M}$ $(=2700 \AA)$, while the thick curve the activation energy predicted in our scheme. The dashed curve represents the SP gap formula. In all these curves we use the parameters $\rho=1.25 \times 10^{11} / \mathrm{cm}^{2}$ and $d=210 \AA$, and their normalization at $\theta=0$ is taken arbitrarily to fit the data in Fig.1. The arrow indicates the critical angle $\Theta_{c}$. 
possessing the smallest energy must be excited thermally, which should be detected as the activation energy. The plasmon formula (5.3) implies that the junction with the maximum size $\ell_{M}$ gives the smallest plasmon energy $E\left(\Theta, \ell_{M}\right)$ in the vicinity of $\Theta=0$.

Let us temporarily assume that the sample contains junctions with two different lengths $\ell$ and $\ell_{M}, \ell<\ell_{M}$. In Fig. 2 we have plotted two curves for the corresponding plasmon energy (5.3) as a function of the tilt angle $\Theta$, using the same values for the sample parameters as in Fig.1. Since the activation energy is given by the smallest plasmon energy, as $\Theta$ increases from $\Theta=0$, it is given by the plasmon energy $E\left(\Theta, \ell_{M}\right)$ up to the critical angle $\Theta_{c}$ where the first minimum is reached. Then, it begins to increases: however, when it crosses the plasmon energy $E(\Theta, \ell)$, it switches to the latter one since $E(\Theta, \ell)<$ $E\left(\Theta, \ell_{M}\right)$. The actual sample contains many junctions with length $\ell$ distributed almost continuously such that $\ell<\ell_{M}$. Then, the activation energy is given by the minimum value of the plasmon energy, i.e., $\Delta_{\text {SAS }}$ for $\Theta>$ $\Theta_{c}$. Hence, if the maximum phase-coherence length is given by $\ell_{M}$, our theoretical activation energy is given by the thick curve in Fig. 2 .

It should be stressed that the flatness of our theoretical curve for $\Theta>\Theta_{c}$ is a direct consequence of the presence of many domains with various size $\ell$, each of which contains plasmon excitations with characteristic frequency. On the other hand, the rapid decrease of the curve in the region $\Theta<\Theta_{c}$ is due to the plasmon excitations in the domains with the maximum size $\ell_{M}$ among many others in the sample.

It is convenient to use the normalized activation energy $E_{A} \equiv E(\Theta) / E(\Theta=0)$ for comparison with the experimental data:

$$
E_{A}(\Theta)=\sqrt{\sin ^{2} \beta\left|\frac{\sin \left(\pi \tan \Theta / \tan \Theta_{c}\right)}{\pi \tan \Theta / \tan \Theta_{c}}\right|+\cos ^{2} \beta}
$$

for $\Theta<\Theta_{c}$ and

$$
E_{A}(\Theta)=\cos \beta
$$

for $\Theta>\Theta_{c}$, where

$$
\tan \beta=\frac{\omega_{J}}{\Delta_{\mathrm{SAS}}},
$$

and

$$
\tan \Theta_{c}=\frac{1}{\ell_{M} d \rho}
$$

The formula is uniquely determined when two parameters $\Theta_{c}$ and $\beta$ are given. Here, since $\Theta_{c}$ involves an unknown parameter $\ell_{M}$, it is to be determined experimentally.

Using our scheme we proceed to analyze the experimental data [16] on the activation energy versus the parallel magnetic field in a DLQH system with $\nu=1$. Experimental data are reported for five different samples, which we have reproduced in Fig.3A $\sim 3 \mathrm{E}$, where the activation energy is normalized by the value at $\Theta=0$. We have also given our theoretical formula (5.4a) and (5.4b) by a thick curve and the SP gap formula (5.1) by a dashed curve. In drawing the curves we have used the measured values of $\Theta_{c}$ and $\beta$ in each samples. The values

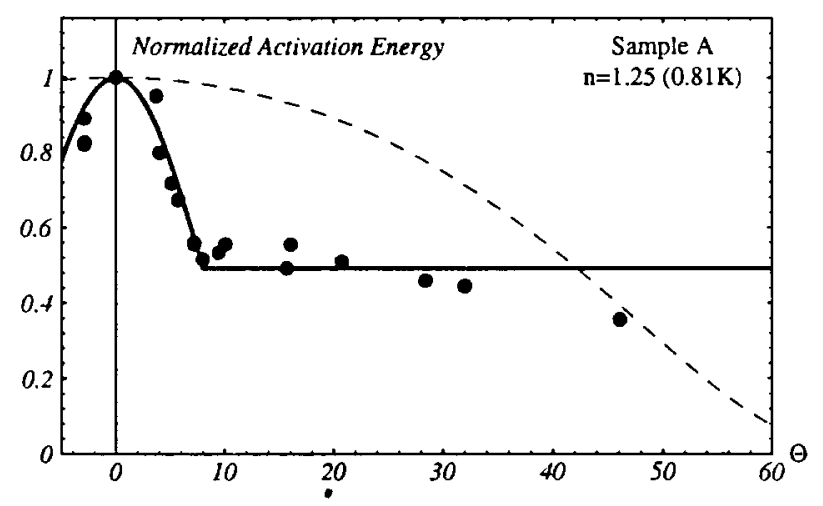

Fig.3A: A fitting of the data by our plasmon formula for sample A $\left(\Delta_{\mathrm{SAS}}=0.81 \mathrm{~K}, \rho=1.25 \times 10^{11} / \mathrm{cm}^{2}\right)$. The dashed curve represents the SP gap formula.

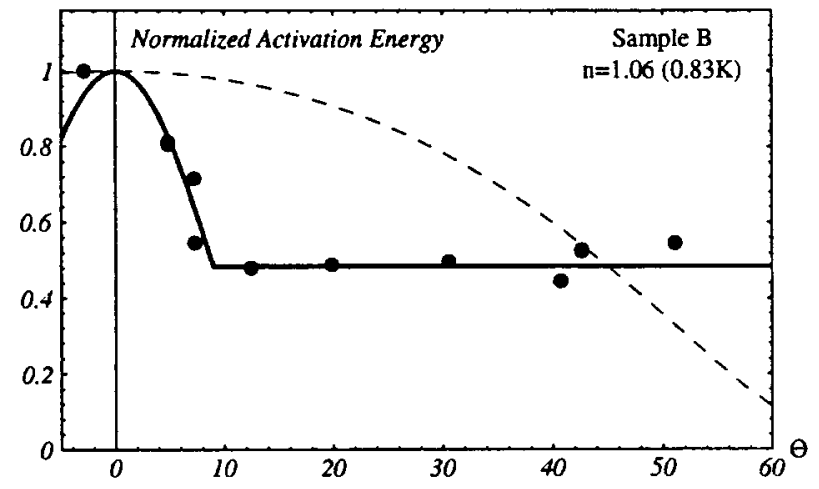

Fig.3B: A fitting of the data by our plasmon formula for sample B $\left(\Delta_{\mathrm{SAS}}=0.83 \mathrm{~K}, \rho=1.06 \times 10^{11} / \mathrm{cm}^{2}\right)$. The dashed curve represents the SP gap formula.

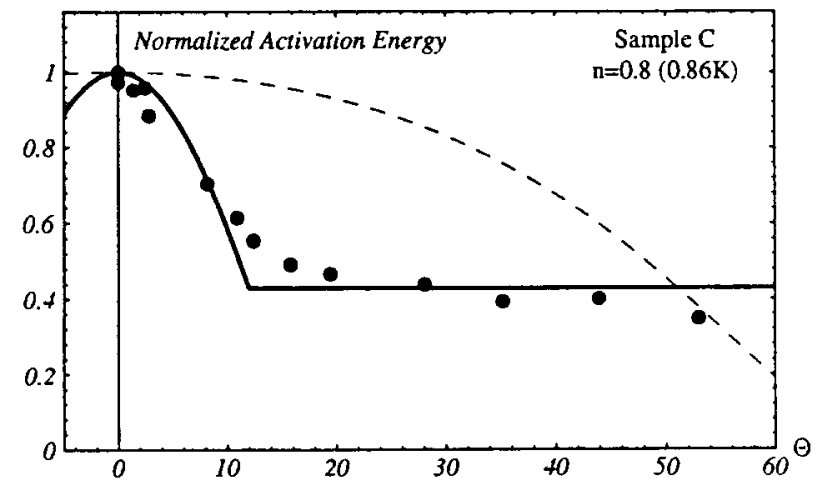

Fig.3C: A fitting of the data by our plasmon formula for sample $\mathrm{C}\left(\Delta_{\mathrm{SAS}}=0.86 \mathrm{~K}, \rho=0.80 \times 10^{11} / \mathrm{cm}^{2}\right)$. The dashed curve represents the SP gap formula. 
of $\Delta_{\mathrm{SAS}}$ and $\rho\left(=n \times 10^{11} / \mathrm{cm}^{2}\right)$ of each sample are summarized in the following table, where we have also given the measured critical value $\Theta_{c}$, and the maximum domain size $\ell_{M}$ calculated by (5.6). The agreements of our theoretical curves and the experimental data are quite good for all these samples.

\begin{tabular}{|c|r|r|r|r|}
\hline sample & $\Delta_{\text {SAS }}$ & $\mathrm{n}$ & $\theta_{\mathrm{c}}$ & $\ell_{M}$ \\
\hline $\mathrm{A}$ & $0.81 \mathrm{~K}$ & 1.25 & $8^{\circ}$ & $2700 \AA$ \\
\hline $\mathrm{B}$ & $0.83 \mathrm{~K}$ & 1.06 & $9^{\circ}$ & $2700 \AA$ \\
\hline $\mathrm{C}$ & $0.86 \mathrm{~K}$ & 0.80 & $12^{\circ}$ & $2700 \AA$ \\
\hline $\mathrm{D}$ & $4.43 \mathrm{~K}$ & 1.20 & $23^{\circ}$ & $930 \AA$ \\
\hline $\mathrm{E}$ & $8.53 \mathrm{~K}$ & 1.21 & $41^{\circ}$ & $450 \AA$ \\
\hline
\end{tabular}

We wish to make some comments on two parameters $\Theta_{c}$ and $\beta$, which we have chosen phenomenologically to fit data in Fig.3. The first comment is on $\Theta_{c}$. Here, $\tan \Theta_{c}$ depends on $\rho$ just as in (5.6). This dependence can be checked to hold experimentally in samples (A), (B) and (C), provided that $\ell_{M}$ is independent of the density $\rho$. Note that this independence is also consistent with the data. As we have stated, the physical picture of the QH-state Josephson junction is that it is made of many tiny domains because the interlayer tunneling acts to prevent the overall phase coherence of the condensate. Since $1 / \Delta_{\text {SAS }}$ is the time scale of one electron staying in one layer, it would be reasonable to speculate that

$$
\ell_{M}=\frac{c_{\ell}}{\Delta_{\mathrm{SAS}}}
$$

with $c_{\ell}$ a numerical constant independent of $\rho$. This anzats is consistent with the following facts. First, in the strong tunneling limit $\left(\Delta_{\text {SAS }} \rightarrow \infty\right)$, the DL system is reduced effectively to a single layer system and there is no Josephson junction $\left(\ell_{M} \rightarrow 0\right)$. (Actually, the collective state would be dissociated into the SP symmetric state when $\ell_{M}$ approaches the magnetic length $\ell_{B}$.) Second, in the weak tunneling limit $\left(\Delta_{\text {SAS }} \rightarrow 0\right.$ ) electrons stay in each wells and form a coherent state with a large phasecoherence length $\left(\ell_{M} \rightarrow \infty\right)$, which are the QH states (3.28). Thus, it is reasonable to assume that in the intermediate strength of the tunneling the phase-coherence length becomes finite. Combining (5.7) with (5.6), we obtain

$$
\tan \Theta_{c}=\frac{\Delta_{\mathrm{SAS}}}{c_{\ell} d \rho}
$$

This linear dependence on $\Delta_{\text {SAS }}$ is qualitatively correct all over the samples, although the predicted slope is 1.5 times larger than the measured values: See the above table and Fig.3 of Ref. [16].

The second comment is on $\beta$, or

$$
\tan \beta=\frac{\omega_{J}}{\Delta_{\mathrm{SAS}}}=\sqrt{\frac{4 \pi e^{2} d \rho_{0}}{\varepsilon \Delta_{\mathrm{SAS}}}},
$$

by using (4.37). Thus, this is a calculable quantity in our scheme. However, its actual value does not agree well with the observed value in each sample, although a rough agreement is obtained in samples (D) and (E) with relatively large $\Delta_{\mathrm{SAS}}(\geq 5 K)$. In samples $(\mathrm{A}),(\mathrm{B})$ and $(\mathrm{C})$ with small $\Delta_{\mathrm{SAS}}\left(\leq 1 \boldsymbol{K}^{-}\right)$the theoretical values of $\tan \beta$ are approximately seven times bigger than the observed ones. At present we have no reasoning why such a significant correction of $\Delta_{\text {SAS }}$ occurs for samples with small $\Delta_{\text {SAS }}$.

We may summarize the physical picture of the activation energy anomaly as follows. (I) As the parallel magnetic field increases, the activation energy decreases rapidly up to the critical angle $\Theta_{c}$. This is because the penetrated magnetic field strongly suppresses the Josephson current. Thus, the plasmon frequency associated with the current decreases rapidly with $\Theta$. (II) As the electron density increases, the critical angle $\Theta_{c}$ becomes smaller, as is confirmed in the table for samples A, B and $C$. This is because the increase of the electron density means the increase of the magnetic field $B_{\perp}$ at $\nu=1$. Hence, the penetrated magnetic flux $\left(\approx B_{\|}=B_{\perp} \tan \Theta\right)$ also increases at fixed $\Theta$, which implies a rapider decrease of the activation energy. (III) An intriguing feature of the

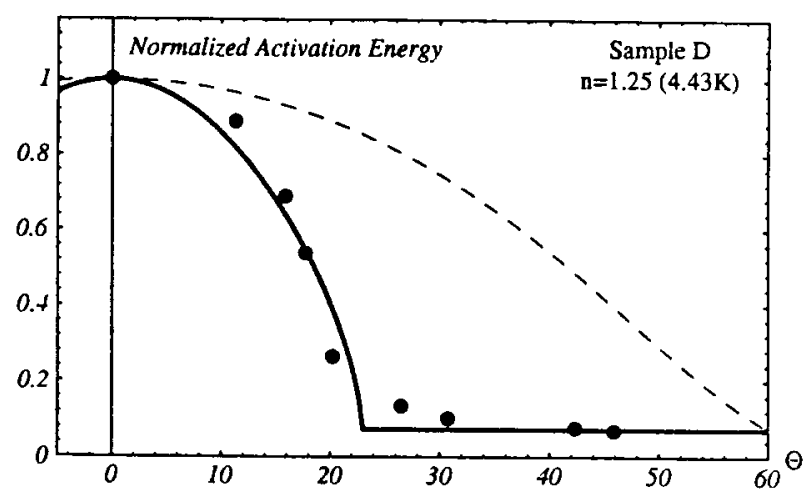

Fig.3D: A fitting of the data by our plasmon formula for sample $\mathrm{D}\left(\Delta_{\mathrm{SAS}}=4.43 \mathrm{~K}, \rho=1.20 \times 10^{11} / \mathrm{cm}^{2}\right)$. The dashed curve represents the SP gap formula.

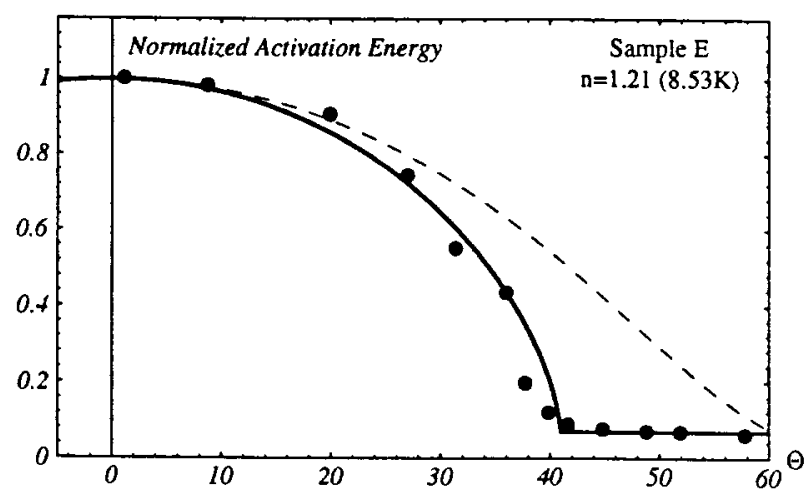

Fig.3E: A fitting of the data by our plasmon formula for sample $\mathrm{E}\left(\Delta_{\mathrm{SAS}}=8.53 \mathrm{~K}, \rho=1.21 \times 10^{11} / \mathrm{cm}^{2}\right)$. The dashed curve represents the SP gap formula. 
experiment data is that the activation energy becomes almost constant for $\Theta>\Theta_{c}$. This constant is given by $\Delta_{\text {SAS }}$ in our scheme, which originates in the tunneling gap energy $E_{\Delta}$ in the Hamiltonian (4.1). As we have mentioned, this constant seems to receive a significant correction especially for small $\Delta_{\mathrm{SAS}}$.

\section{B. Proposal of new experiments}

It is a fascinating idea that Anderson plasmons may have already been observed experimentally. To confirm this it is necessary to do experiments to observe plasmon excitations more directly. In our picture the flatness of the activation energy for $\Theta>\Theta_{c}$ originates in the existence of many domains with their typical scale being $\sim 1000 \AA$. Therefore, if experiments can be done on a sample with the scale being several thousand $\stackrel{\AA}{A}$, we expect to observe an interference-like pattern in the activation energy as a function of the tilt angle. This is because the sample contains only several domains. If it contains just one domain, the activation energy is just given by the plasmon formula (5.3) with $\ell$ being the size of the domain. Thus, the interference-like pattern will manifest itself more clearly as the sample becomes smaller.

It is also important to do microwave experiments. The key point is that Anderson plasmons are massive photons resulting from their coupling with the charging $e \Delta \rho$, and hence they must be excited by microwave. By applying microwave, the plasmons are excited and then they decay with the emission of photons. Such an experiment has been carried out and the Anderson plasmon has been observed in superconductor Josephson junction [22]. There are distinctive features in the case of the $\mathrm{QH}$ state because of the domain structure in the sample.

In the absence of the parallel magnetic field $\left(B_{\|}=0\right)$, the frequency spectrum of the plasmons is the same in all domains, where the plasmon frequency is given by (4.36) or

$$
\omega_{P}^{0}=\sqrt{\omega_{J}^{2}+\Delta_{\mathrm{SAS}}^{2}}
$$

However, in the existence of the parallel magnetic field $\left(B_{\|} \neq 0\right)$, the spectrum is different in each domain depending on the size $\ell$ of the domain, that is, the plasmon frequency is given as a function of the tilt angle $\Theta$ and the size $\ell$ as in (4.45) or

$$
\omega_{P}(\Theta, \ell)=\sqrt{\omega_{J}^{2}\left|\frac{\sin \left(2 \pi d \ell \rho_{0} \tan \Theta\right)}{2 \pi d \ell \rho_{0} \tan \Theta}\right|+\Delta_{\mathrm{SAS}}^{2}}
$$

These plasmons are excited and emit photons (microwave) when appropriate microwave is applied externally.

For the sake of simplicity let us assume that there are no other excitations other than Anderson plasmons in the frequency range we are interested in $\left(\omega_{P} \approx 10 K^{-}\right)$. At a fixed tilt angle $\Theta$ we apply microwave with frequency $\omega$ with a fixed intensity, starting with $\omega=0$. There will be no microwave radiation from the sample until it reaches $\omega_{P}\left(\Theta, \ell_{M}\right)$ as far as $\Theta<\Theta_{c}$, where the plasmons with this frequency is excited and emit microwave of the same frequency. The sample will continue to emit microwave until the frequency $\omega$ of the external microwave passes over the frequency $\omega_{P}\left(\Theta, \ell_{\min }\right)$, with $\ell_{\min }$ the minimum size of the domains in the sample: After that there is no microwave emission from the sample because there are no plasmons whose energy $\omega_{P}$ is larger than $\omega_{P}\left(\Theta, \ell_{\min }\right)$. Hence, the microwave emission occurs in the frequency range of $\omega_{P}\left(\Theta, \ell_{M}\right)<\omega<\omega_{P}\left(\Theta, \ell_{\min }\right)$ as far as $\Theta<\Theta_{c}$.

What is the intensity of this emitted microwave ? It depends on the number of domains in the sample emitting the plasmon with the frequency in problem. This can only be answered when the distribution $f(\ell)$ of the domain with the size $\ell$ is known. It is given by the formula,

$$
\begin{aligned}
\text { Intensity } & =\int_{\ell_{\min }}^{\ell_{M}} d \ell f(\ell) \delta\left[\omega-\omega_{P}(\Theta, \dot{\ell})\right] \\
& =\frac{f\left(\ell_{0}\right)}{\left|\partial \omega_{P}\left(\Theta, \ell_{0}\right) / \partial \ell\right|},
\end{aligned}
$$

with $\ell_{0}$ satisfying $\omega=\omega_{P}\left(\Theta, \ell_{0}\right)$. We can estimate this formula in the vicinity of $\Theta=0$. Obviously the intensity of the microwave emission is the strongest at $\Theta=0$, because all the domains emit the plasmons with the same frequency $\omega_{P}^{0} \equiv \omega_{P}\left(\Theta=0, \ell_{M}\right)$. As far as $\Theta$ is small enough, we may approximate as $\ell_{0}=\ell_{M}$ in $f\left(\ell_{0}\right)$ because the distribution will be smooth around $\ell=\ell_{M}$. Furthermore, we may expand as

$$
\omega_{P}(\Theta, \ell) \approx \omega_{P}^{0}-\frac{\left(\omega_{\mathrm{J}} \pi d \ell \rho_{0} \Theta\right)^{2}}{3 \omega_{P}^{0}} .
$$

Then, for $\Theta \ll 1$ the intensity formula (5.12) is evaluated as

$$
\text { Intensity }=\frac{f\left(\ell_{M}\right)}{2 \pi \omega_{J} d \rho_{0} \Theta} \sqrt{\frac{3 \omega_{P}^{0}}{\omega_{P}^{0}-\omega}}
$$

where $\omega_{P}\left(\Theta, \ell_{M}\right)<\omega<\omega_{P}\left(\Theta, \ell_{\min }\right)$. These features of the emitted microwave intensity are characteristic to the QH state with the domain structure. Hence, the observation of this type of microwave emission is a strong support of our theory of the QH states. Furthermore, we expect an interference pattern to appear as in superconductor Josephson junction [22] when microwave experiments are done on a sufficiently tiny sample with small value of $\Delta_{\mathrm{SAS}}$.

\section{DISCUSSIONS}

In this paper we have explained how the Josephson phenomena are possible in certain DLQH systems. The 
essence is the existence of degenerate QH states possessing various electron number difference $\Delta N$ between the two layers. Once a weak tunneling of electrons is allowed between the layers, these $\mathrm{QH}$ states are reorganized and specified by the phase $\theta$, as in superconductor Josepshon junction, The Josephson effect in $\mathrm{QH}$ states must yield the characteristic feature of the condensation of unpaired electrons, presenting a direct demonstration of statistics transmutation on the plane. This statistical transformation is widely believed for understanding fractional $\mathrm{QH}$ states. In this way, the QH-state Josephson junction has a capability to open an entirely new branch of the condensed matter physics.

We have also interpreted our results in terms of the pseudospin language. In so doing we have gained some insights on the physical picture of the Josephson phenomena.

Experimental verifications of these phenomena are most important. We have pointed out an intriguing possibility that a recent experiment [16] has already observed Anderson plasmons intrinsic to the QH-state Josephson junction. We have also proposed a microwave experiment without attaching external leads for a direct observation of these plasmons.

\section{ACKNOWLEDGMENTS}

We are very grateful to Sheena Murphy for valuable correspondence and for allowing us to reproduce their experimental data in our figures. A.I. appreciates the hospitality of the staff members at Institute for Nuclear Study, University of Tokyo, where a part of this work was done. This work was supported in part by Grantin-Aid for Scientific Research from the Ministry of Education, Science and Culture (No.05640320) and MultiDisciplinary Science Foundation.

[1] The Quantum Hall Effect, edited by S. Girvin and R. Prange (Springer-Verlag, New York, 1990), 2nd ed.

[2] G.S. Boebinger, H.W. Jiang, L.N. Pfeiffer and K.W. West, Phys. Rev. Lett. 64, 1793 (1990); Y.W. Suen, L.W. Engel, M.B. Santos, M.Shayegan, and D.C. Tsui, Phys. Rev. Lett. 68, 1379 (1992); J.P. Eisenstein, G.S. Boebinger, L.N. Pfeiffer, K.W. West and Song He, Phys. Rev. Lett. 68, 1383 (1992).

[3] Z.F. Ezawa and A. Iwazaki, Phys. Rev. B 47, 7295 (1993).

[4] Z.F. Ezawa and A. Iwazaki, Phys. Rev. Lett. 70, 3119 (1993).
[5] Z.F. Ezawa and A. Iwazaki, Phys. Rev. B 48, 15189 (1993).

[6] Z.F. Ezawa and A. Iwazaki, "Josephson Effect and Anderson Plasmons in Double-Layer Quantum Hall System" preprint TU-447 (October 1993).

[7] Z.F. Ezawa, A. Iwazaki and Y.S. Wu, Mod. Phys. Lett. B 7, 1223 (1993); 7, 1825 (1993).

[8] X.G. Wen and A. Zee, Phys. Rev. B 47, 2265 (1993).

[9] D. Finkelstein and J. Rubinstein, J. Math. Phys. 9, 1762 (1968); J.M. Leinaas and J. Myrheim, Nuovo Cim. 37B, 1 (1977).

[10] S.M. Girvin and A.H. MacDonald, Phys. Rev. Lett. 58, 1252 (1987); S.C. Zhang, T.H. Hansen and S. Kivelson, Phys. Rev. Lett. 62, 82 (1989); N. Read, Phys. Rev. Lett. 62, 86 (1989); Z.F. Ezawa and A. Iwazaki, Phys. Rev. B 43, 2637 (1991).

[11] B.I. Halperin, Helv. Phys. Acta 56, 75 (1983).

[12] See, e.g., Introduction to Superconductivity, M. Tinkham (McGraw-Hill, New York, 1975); Physics and Applications of the Josephson Effect, A. Barone and G. Paterno (J. Wiley Sons, New York, 1982).

[13] A.H. MacDonald and S.C. Zhang, preprint "Collective excitations in double-layer quantum Hall systems"; $K$. Yang, K. Moon, L. Zheng, A.H. MacDonald, S.M. Girvin, D. Yoshioka and S.C. Zhang, Phys. Rev. Lett. 72, 732 (1994).

[14] Z.F. Ezawa, M. Hotta and A. Iwazaki, Phys. Rev. B 46, 7765 (1992); Z.F. Ezawa and A. Iwazaki, J. Phys. Soc. Jpn 61, 4133 (1992).

[15] H. A. Fertig, Phys. Rev. B 40, 1087 (1989); A.H. MacDonald, P.M. Platzman and G.S. Boebinger, Phys. Rev. Lett. 65, 775 (1990).

[16] S.Q. Murphy, J.P. Eisenstein, G.S. Boebinger, L.N. Pfeiffer and K.W. West, Phys. Rev. Lett. 72, 728 (1994).

[17] Y. Aharonov and D. Bohm, Phys. Rev. 115, 485 (1959).

[18] R. Jackiw and S-Y. Pi, Phys. Rev. D 42, 3500 (1990).

[19] E.B. Bogomol'nyi, Sov. J. Nucl. Phys. 24, 449 (1976).

[20] S.M. Girvin, A.H. MacDonald, M.P.A. Fisher, S-J. Rey and J.P. Sethna, Phys. Rev. Lett. 65, 1671 (1990).

[21] M. Rasolt, F. Perrot and A.H. MacDonald, Phys. Rev. Lett. 55, 433 (1985); M. Rasolt and A.H. MacDonald, Phys. Rev. B 34, 5530 (1986); X.G. Wen and A. Zee, Phys. Rev. Lett. 69, 1811 (1992); Z.F. Ezawa and A. Iwazaki, Int. J. Mod. Phys. B 6, 3205 (1992).

[22] A.J. Dahm, A. Denenstein, T.F. Finnegan, D.N. Langenberg and D.J. Scalapino, Phys. Rev. Lett. 20 $859 ; 1020$ (E) (1968). 
